\title{
Functional Genomic Analysis of Oligodendrocyte Differentiation
}

\author{
Jason C. Dugas, ${ }^{1}$ Yu Chuan Tai, ${ }^{3}$ Terence P. Speed, ${ }^{2,5}$ John Ngai, ${ }^{4,5}$ and Ben A. Barres ${ }^{1}$ \\ ${ }^{1}$ Department of Neurobiology, Stanford University School of Medicine, Stanford, California 94305, and ${ }^{2}$ Department of Statistics, ${ }^{3}$ Program in Biostatistics, \\ ${ }^{4}$ Department of Molecular and Cell Biology and Helen Wills Neuroscience Institute, and ${ }^{5}$ Functional Genomics Laboratory, University of California, \\ Berkeley, California 94720
}

To better understand the molecular mechanisms governing oligodendrocyte (OL) differentiation, we have used gene profiling to quantitatively analyze gene expression in synchronously differentiating OLs generated from pure oligodendrocyte precursor cells in vitro. By comparing gene expression in these OLs to OLs generated in vivo, we discovered that the program of OL differentiation can progress normally in the absence of heterologous cell-cell interactions. In addition, we found that OL differentiation was unexpectedly prolonged and occurred in at least two sequential stages, each characterized by changes in distinct complements of transcription factors and myelin proteins. By disrupting the normal dynamic expression patterns of transcription factors regulated during OL differentiation, we demonstrated that these sequential stages of gene expression can be independently controlled. We also uncovered several genes previously uncharacterized in OLs that encode transmembrane, secreted, and cytoskeletal proteins that are as highly upregulated as myelin genes during OL differentiation. Last, by comparing genomic loci associated with inherited increased risk of multiple sclerosis (MS) to genes regulated during OL differentiation, we identified several new positional candidate genes that may contribute to MS susceptibility. These findings reveal a previously unexpected complexity to $\mathrm{OL}$ differentiation and suggest that an intrinsic program governs successive phases of OL differentiation as these cells extend and align their processes, ensheathe, and ultimately myelinate axons.

Key words: oligodendrocyte; genomics; Affymetrix; myelin; multiple sclerosis; differentiation

\section{Introduction}

We are interested in understanding the development and function of oligodendrocytes (OLs), the myelin-forming glial cells of the CNS. OLs are generated by oligodendrocyte precursor cells (OPCs), which are derived from precursors in germinal areas of the CNS (Rowitch, 2004). OPCs then migrate and proliferate throughout the CNS before differentiating into myelinating OLs. In addition to forming myelin sheaths to enhance nerve conduction velocity, OLs serve other important functions: they play a crucial role in organizing axonal voltage-dependent $\mathrm{Na}^{+}$and $\mathrm{K}^{+}$ channels, induce a profound increase in myelinated axon diameter, help buffer $\mathrm{K}^{+}$ions released by axons, and provide neurotrophic support (Baumann and Pham-Dinh, 2001).

The molecular mechanisms that control OL development and

\footnotetext{
Received June 19, 2006; revised Sept. 6, 2006; accepted Sept. 11, 2006.

This work was supported by the Myelin Repair Foundation (B.A.B.); the Wadsworth Foundation (B.A.B.); National Eye Institute-National Institutes of Health (NIH) Grant R01 EY10257; NIH Grants HG0047 (Y.C.T.), R01 LM07609-01 (T.P.S.), R01 MH61665 (J.N.), and R01 DC007235 (J.N.); and National Multiple Sclerosis Society Grant RG2332 (B.A.B.). We thank Guy H. Fernald, Sergio E. Baranzini, and Stephen L. Hauser for generously allowing us access to their Multiple Sclerosis linkage analyses study before publication, which was used in the MS loci-regulated gene correlation analysis described in this work.

Correspondence should be addressed to Dr. Jason C. Dugas, Department of Neurobiology, Stanford University School of Medicine, Fairchild Building Room D206, 299 Campus Drive, Stanford, CA 94305-5125. E-mail: jcdugas@alum.mit.edu.

Y. C. Tai's present address: The Sidney Kimmel Comprehensive Cancer Center, Johns Hopkins School of Medicine, Baltimore, MD 21205.

DOI:10.1523/JNEUROSCI.2572-06.2006

Copyright $\odot 2006$ Society for Neuroscience $\quad$ 0270-6474/06/2610967-17\$15.00/0
}

function are still poorly understood. Several transcription factors have been identified that are necessary for OPC and OL generation. These include SOX10, OLIG1, and OLIG2, and homeodomain proteins of the Nkx family (Wegner, 2001; Stolt et al., 2002; Zhou and Anderson, 2002; Masahira et al., 2006; Nakamura et al., 2006; Wu et al., 2006). These various transcription factors are expressed by both OPCs and OLs, and mutations in some of these genes lead to the loss of OPCs as well as OLs. It remains unclear whether these transcription factors are sufficient to govern the final stages of OL differentiation, or whether additional transcription factors or other gene expression changes are required.

Genetic screens using invertebrate model systems have been a powerful approach for understanding the molecular basis of neuronal specification, but because OLs are mostly a vertebrate adaptation we know much less about their generation. An alternative to genetic screening is to use recently developed gene profiling methods. Previous gene profiling studies of OPCs and OLs have focused on either the process of demyelination versus remyelination in CNS tissue derived from cuprizonedemyelinated animals (Jurevics et al., 2002; Arnett et al., 2003), or on the differences between uncommitted neural precursors and OPCs (Hu et al., 2004), as opposed to the terminal differentiation step of primary OPCs into mature OLs.

In the present study, we have used our ability to highly purify and culture primary OPCs from the developing CNS (Barres et al., 1992, 1993) to elucidate the gene expression changes occurring specifically during the OPC to OL transition. Using this 
preparation, we have addressed the issue of whether OL differentiation is a rapid, single event or a prolonged, multistep process. Previous studies have found that OPCs differentiate relatively rapidly (2-3 d) into OLs expressing myelin basic protein, proteolipid protein, and other major myelin proteins, but a few OLspecific genes, such as myelin oligodendrocyte glycoprotein and myelin-associated oligodendrocyte basic protein, are specifically induced several days later (Holz and Schwab, 1997; Baumann and Pham-Dinh, 2001). It remains unknown whether these lateexpressed genes are indicative of a distinct phase of OL differentiation, or simply represent a small subset of genes upregulated by axonal contact or other environmental cues during the final stages of myelination. Our ability to purify and induce the differentiation of OPCs provided us with the unique opportunity to observe in fine temporal detail the intrinsic differentiation program of a homogenous population of isolated, fate-restricted mammalian cells.

Using Affymetrix microarrays to analyze gene expression during synchronous OL differentiation from OPCs in vitro, we were able to discern that this process is unexpectedly prolonged and occurs in distinct temporal stages, each of which are indicated by the induction and repression of multiple genes. By disrupting the normal expression patterns of transcription factors linked to these separate stages, we have demonstrated that genes induced early and late during OL differentiation can be independently regulated. Furthermore, similar patterns of gene expression observed in OLs generated in vitro and in vivo indicate that the complete program of normal OL differentiation can take place in the absence of heterologous cell-cell interactions. Finally, by comparing genomic loci associated with inherited increased risk of multiple sclerosis (MS) to genes regulated during OL differentiation, we have identified several new candidate genes for MS susceptibility.

\section{Materials and Methods}

Detailed protocols are available on request from B. A. Barres at barres@stanford.edu.

Cell purification. Cortical OPCs and OLs were purified by sequential immunopanning as described previously (Chan et al., 2004). Briefly, brains were obtained from postnatal day 7 (P7) (for OPCs) or P10-P12 (for OLs) Sprague Dawley rats (Charles River, Wilmington, MA), and the cerebellum and midbrain/hindbrain structures were removed by crude dissection. Tissue was diced and digested in papain (Worthington, Lakewood, $\mathrm{NJ}$ ) at $37^{\circ} \mathrm{C}$ for $90 \mathrm{~min}$, and then gently dissociated. Dissociated P7 brains were sequentially immunopanned on Ran-2, antigalactocerebrosidase ( $\mathrm{GalC}$ ), and then $\mathrm{O} 4$ antibody-coated plates to select $\mathrm{GalC}^{-} \mathrm{O}_{4}^{+}$OPCs. Dissociated P10-P12 brains were sequentially immunopanned on Ran-2, $2 \times \mathrm{A} 2 \mathrm{~B} 5$, and then anti-GalC antibodycoated plates to select $\mathrm{A} 2 \mathrm{~B} 5{ }^{-} \mathrm{GalC}^{+}$OLs. After rinsing nonadherent cells away, acutely purified OLs or OPCs were incubated directly on the final panning plate in differentiation or proliferation promoting medium, respectively, for $30 \mathrm{~min}$ at $37^{\circ} \mathrm{C}, 10 \% \mathrm{CO}_{2}$ to allow for brief recovery of gene expression before harvesting cells for RNA. Purified OPCs to be cultured were removed from the final panning plate with trypsin (Sigma, St. Louis, MO) and transferred to poly-D-lysine (pDL) (Sigma)coated tissue culture dishes or $12 \mathrm{~mm}$ glass coverslips in 24-well tissue culture plates containing proliferation medium.

Cell culture. All cells were cultured at $37^{\circ} \mathrm{C}, 10 \% \mathrm{CO}_{2}$ in DMEM (Invitrogen, Carlsbad, CA) containing human transferrin $(100 \mu \mathrm{g} / \mathrm{ml})$, bovine serum albumin $(100 \mu \mathrm{g} / \mathrm{ml})$, putrescine $(16 \mu \mathrm{g} / \mathrm{ml})$, progesterone (60 ng/ml), sodium selenite (40 ng/ml), $N$-acetyl-L-cysteine $(5 \mu \mathrm{g} / \mathrm{ml})$, D-biotin $(10 \mathrm{ng} / \mathrm{ml})$, forskolin $(4.2 \mu \mathrm{g} / \mathrm{ml})$, bovine insulin $(5 \mu \mathrm{g} / \mathrm{ml})($ all from Sigma), glutamine $(2 \mathrm{~mm})$, sodium pyruvate $(1 \mathrm{~mm})$, penicillinstreptomycin (100 U each) (all from Invitrogen), Trace Elements B (1×; Mediatech, Herndon, VA), and CNTF (10 ng/ml; gift from Regeneron,
Tarrytown, NJ). Proliferation medium also contained OPC mitogens PDGF-AA (10 ng/ml) and NT-3 (1 ng/ml) (both from PeproTech, Rocky Hill, NJ); differentiation medium also contained triiodothyronine (T3) (40 ng/ml; Sigma) without OPC mitogens.

RNA purification and gene chip hybridization. Total RNA was isolated from acutely purified or cultured cells with the RNeasy mini kit (Qiagen, Valencia, CA), using Qiashredder columns for cell lysis, and inserting Qiagen on-column DNase steps to remove any contaminating genomic DNA. For each sample, 1-2 $\mu \mathrm{g}$ of total RNA was amplified according to protocols recommended by Affymetrix (Santa Clara, CA). Briefly, a poly-T primer containing a T7 RNA polymerase site was used to generate first-strand cDNA. After subsequent second-strand cDNA generation, RNA was generated using the Ambion (Austin, TX) T7 MegaScript kit. One microgram of each first-round amplified cRNA sample was amplified a second time, using random hexamers to prime first-strand cDNA generation, and reincorporating T7-poly-T primers during secondstrand cDNA generation. Biotin-labeled cRNA was then generated from the entire quantity of each second-round generated cDNA sample (ENZO BioArray HighYield RNA Transcript Labeling kit; ENZO, Farmingdale, NY), fragmented, and applied to Affymetrix rat genomic U34A-C chips at the Stanford Protein and Nucleic Acid Biotechnology Facility according to Affymetrix protocols.

Data analysis. CEL files obtained via MAS 5.0 (Affymetrix) were analyzed in GeneTraffic 3.2 (Stratagene, La Jolla, CA), using the robust multichip analysis (RMA) method to obtain individual probe set expression values (Irizarry et al., 2003). For analyses of gene expression changes, the baseline value for each individual probe set was set as the arithmetic mean of the expression values of the four OPC replicate samples on the corresponding probe set. Fold expression level changes relative to average OPC levels (individual expression level/average OPC expression level) were calculated for each individual biological replicate time point sample and expressed on a $\log _{2}$ scale. The four $\log _{2}$ (fold expression level changes) for each distinct time point were then averaged to obtain the geometric mean of the expression level changes (subsequently referred to as "OPC normalized $\log _{2}$ expression"). Statistical analyses described in Results were performed on OPC normalized $\log _{2}$ expression values. To obtain "raw" expression values presented for each time point (supplemental Table S2, available at www.jneurosci.org as supplemental material; etc.), OPC normalized $\log _{2}$ expression values were anti-logged, and multiplied by the baseline average OPC expression value. Present/marginal/absent calls for individual probe sets were obtained by comparing perfect match to mismatch probe set intensities in MAS 5.0. Mouse and human homologs to the rat genes represented by Affymetrix probe sets were identified either in HomoloGene when data were available, or by BLAST searching with sequences corresponding to rat Affymetrix probe sets when not present in HomoloGene (http://www.ncbi.nlm.nih.gov/). Information on genomic localization was also obtained from the $\mathrm{Na}-$ tional Center for Biotechnology Information (NCBI) database. Functional gene classifications were derived from Gene Ontology information found in NCBI and Stanford SOURCE databases (http://genomewww5.stanford.edu), and from independent literature mining. To determine in situ expression patterns for selected genes, we consulted the Allen Brain Atlas (http://www.brain-map.org/), Brain Gene Expression Map (http://www.stjudebgem.org/), and GENSAT (NCBI) databases.

Comparative RT-PCR. IntraSpec Comparative RT-PCR was performed according to Ambion protocols. Briefly, a first RNA sample is reverse-transcribed with a poly- $\mathrm{T}$ primer that has an additional specific sequence "tag" 10 bp upstream of the poly-T region. A second RNA sample is similarly reverse-transcribed with a poly-T primer containing the same specific primer "tag" 50 bp upstream of the poly-T. Subsequently, when the two samples are mixed together in equal amounts (as determined by radioisotope incorporation into cDNA) and amplified with a single PCR primer pair (a 5' gene-specific primer, and a $3^{\prime}$ primer to the "tag" added downstream of the poly-A tail during cDNA generation), two products different by $40 \mathrm{bp}$ are generated, corresponding to relative amounts of the target gene in the two original samples. RNA was obtained from acutely purified OPCs and OLs, two separate preps for each, distinct from preps used for gene chip hybridizations. In the first pair, OPC cDNA was generated with the short primer, and OL cDNA 


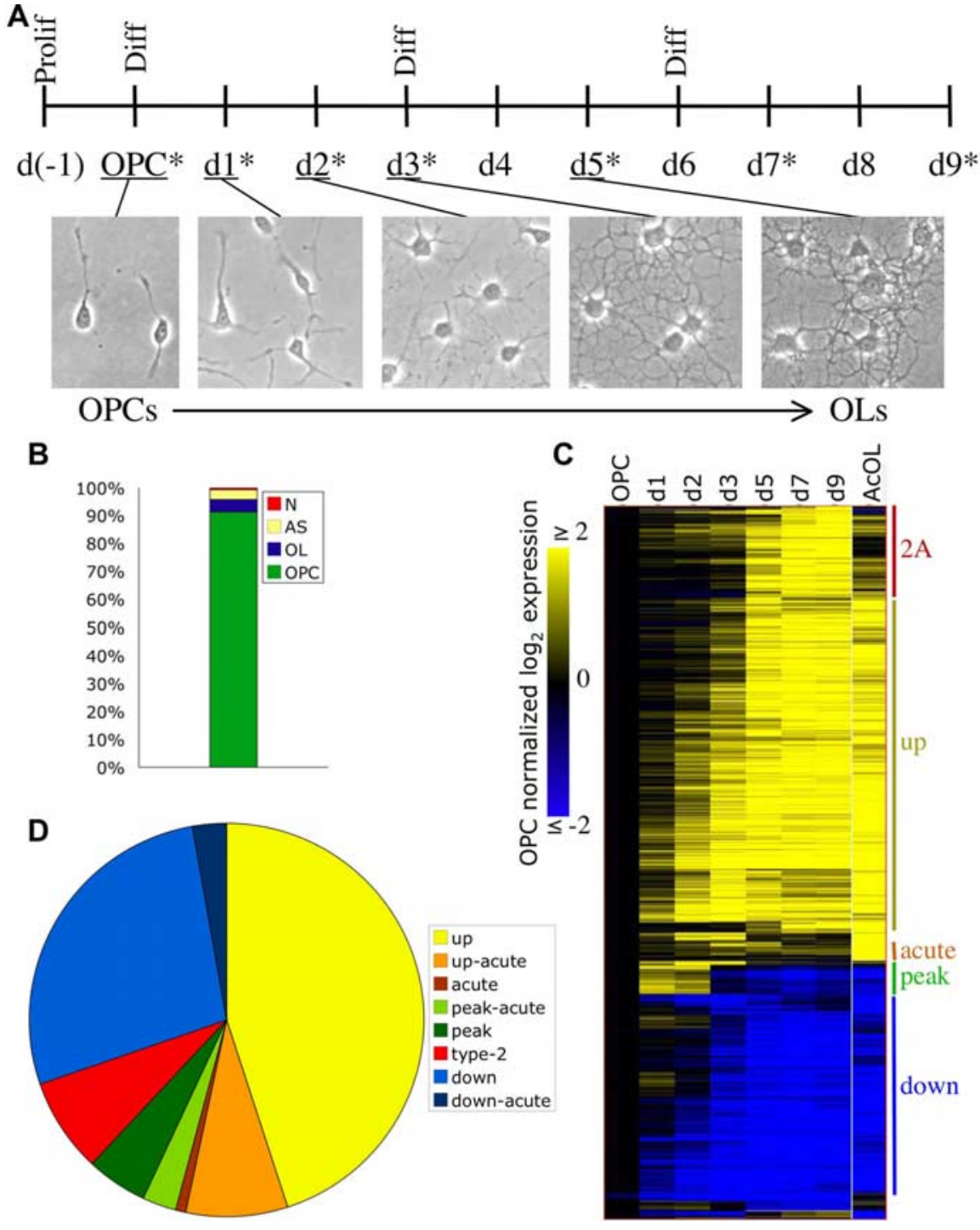

Figure 1. Expression patterns of genes regulated during $0 \mathrm{~L}$ differentiation. $A, O \mathrm{PC}$ s purified from $\mathrm{P} 7$ rat brains were plated into proliferation medium (prolif) in several parallel dishes. At $24 \mathrm{~h}$ after plating (day 0 , "OPC"), cultures were switched entirely into differentiation medium (diff). Remaining cells were fed differentiation medium at days 4 and 7 . Asterisks ${ }^{*}$ ) correspond to time points at which RNA samples were collected; the "OPC" sample was taken from cells never exposed to differentiation medium. Below the time line are phase contrast pictures of cultured OPCs as they differentiate into OLs at indicated time points. No additional morphological changes are seen past day 5 in differentiation medium. $\boldsymbol{B}$, The purity of the initial $\mathrm{OPC}$ cultures was assessed after $24 \mathrm{~h}$ in proliferation medium. Cultures were stained for NG2 (OPC, 91.3\%), GC (OL, 4.5\%), GFAP [astrocytes (AS), 3.4\%], and neurofilament [neurons(N), 0.7\%]. Note that only the NG2 ${ }^{+}$cells appeared fully healthy at this time point (data not shown). C, Expression patterns of the 953 selected highly regulated Affymetrix probe sets. Each horizontal line in the figure corresponds to one selected probe set, with average expression levels at each of the indicated time points illustrated in the eight corresponding columns; $\mathrm{AcOL}$, acutely purified $0 \mathrm{~L}$. For each probe set, expression values depicted are changes relative to the starting average OPC expression level, expressed on a $\log _{2}$ scale. All changes $\geq 4 \times$ up $\left(\geq \log _{2} 2\right)$ are strongest yellow, and all changes $\geq 4 \times$ down $\left(\leq \log _{2}-2\right)$ are strongest blue. Gene expression patterns were grouped using the average hierarchical clustering method with a Pearson correlation as the distance metric in GeneTraffic (Stratagene); similar expression patterns are labeled: probable type 2 astrocyte in red, upregulated in yellow, strongly upregulated only in acutely purified $\mathrm{OLs}$ in orange, early expression peak followed by downregulation in green, and downregulation in blue. $\boldsymbol{D}$, Cumulative expression pattern frequency for the selected regulated probe sets. Individual probe set expression patterns are listed in supplemental Table S2 (available at www.jneurosci.org as supplemental material). Here, "down" also includes probe sets that appear transiently downregulated but also lower in acute $0 \mathrm{~L}$ samples ("dip-down" on supplemental Table S2, available at www.jneurosci.org as supplemental material), and "peak" includes probe sets that peak and then drop to levels lower than OPCs ("peak-down" on supplemental Table S2, available at www.jneurosci.org as supplemental material). The percentages for each expression pattern are as follows: up, 45.12\%; up-acute, $8.18 \%$; acute, $0.73 \%$; peak-acute, 3.04\%; peak, 4.93\%; type 2, 7.66\%; down, 27.6\%; down-acute, $2.73 \%$.

with the long primer. In the second pair, the cDNA primers were reversed.

Immunostaining. Purified OPCs were plated onto pDL-coated $12 \mathrm{~mm}$ glass coverslips in 24-well tissue culture plates and cultured similarly to the method used to generate RNA samples for the time course (Fig. $1 \mathrm{~A}$ ). Coverslips were fixed in $4 \%$ paraformaldehyde for $10 \mathrm{~min}$ at $25^{\circ} \mathrm{C}$, and then incubated for $1 \mathrm{~h}$ in a $50 \%$ goat serum, $1 \%$ bovine serum albumin (BSA), and $100 \mathrm{~mm}$ L-lysine solution to block nonspecific binding. Samples to be stained for cyclic nucleotide phosphodiesterase 1 (CNP1), myelin basic protein (MBP), GFAP, or neurofilament (NF) were permeabilized by the addition of $0.4 \%$ Triton X-100 to the blocking buffer, samples stained for myelin oligodendrocyte glycoprotein (MOG) were permeabilized with $0.05 \%$ Triton X-100, samples to be stained for NG2 chondroitin sulfate proteoglycan (NG2) or galactocerebroside (GC) were not permeabilized. Coverslips were then incubated overnight at $25^{\circ} \mathrm{C}$ in $10 \%$ goat serum, $1 \%$ BSA, $100 \mathrm{~mm}$ L-lysine solution containing either 1:50 anti-MOG antibodies (mouse monoclonal hybridoma supernatant, from R. Reynolds, Imperial College, London, UK), 1:100 anti-CNP1 (MAB326; Chemicon, Temecula, CA), 1:100 antiMBP (ab7349; AbCam, Cambridge, MA), 1:2000 anti-GFAP (G3893; Sigma), 1:10,000 antineurofilament heavy chain (cocktail; Sternberger SMI-31 plus SMI-32; Covance Research Products, Berkeley, CA), 1:400 anti-NG2 (AB5320; Chemicon), or anti-GC (Ranscht et al., 1982); CNP1, MBP, GFAP, and NF staining plus $0.08 \%$ Triton X-100. Transfection experiments were costained with 1:2000 anti-GFP antibodies (AB16901; Chemicon). Finally, coverslips were incubated for $1 \mathrm{~h}$ at $25^{\circ} \mathrm{C}$ in $1 \%$ BSA, $100 \mathrm{~mm}$ L-lysine solution containing the following: antiMOG, -CNP1, -GFAP, -NF, and -GC (1:500) Alexa Fluor 594 goat anti-mouse secondary antibody (A-11032; Invitrogen); anti-NG-2 (1:500) Alexa Fluor 488 goat anti-rabbit (A-11034; Invitrogen); anti-MBP (1:500) Alexa Fluor 594 goat anti-rat (A-11007; Invitrogen); anti-GFP (1:500) Alexa Fluor 488 goat anti-chicken (A-11039; Invitrogen), and then mounted in Vectashield plus 4',6' -diamidino-2-phenylindole (Vector Laboratories, Burlingame, CA) to allow identification of healthy cell nuclei.

Western blots. Optic nerves were dissected from P3, P19, and adult ( $>$ P60) Sprague Dawley rats and lysed with a Dounce tissue homogenizer in radioimmunoprecipitation assay buffer containing Complete Protease Inhibitor Cocktail (Roche, Indianapolis, IN). Standard BCA assays were used to quantify the amount of protein present, and $4 \mu \mathrm{g}$ of each sample was boiled for $5 \mathrm{~min}$ in sample buffer before loading onto a $4-15 \%$ polyacrylamide Ready Gel (BioRad, Hercules, CA), along with secreted, purified transgenic human pancreatic lipase (generously provided by M. Lowe, Children's Hospital, Pittsburgh, PA). Gels were transferred to Immobilon-P membranes (Millipore, Billerica, MA) and, after blocking in 5\% milk, $0.1 \%$ Triton in PBS for $1 \mathrm{~h}$ at $25^{\circ} \mathrm{C}$, were incubated with 1:2000 anti-pancreatic lipase (rabbit serum; M. Lowe) or $1 / 5000$ anti- $\beta$-actin (mouse monoclonal; Sigma) overnight at $4^{\circ} \mathrm{C}$. Blots were then incubated for $1 \mathrm{~h}$ at $25^{\circ} \mathrm{C}$ in HRP-conjugated goat anti-rabbit or goat anti-mouse (Chemicon) and developed with Amersham Biosciences (Piscataway, NJ) Detection Reagent $1+2$. 
Transfections. OPCs were purified from P7 Sprague Dawley rat brains and cultured for $6 \mathrm{~d}$ in proliferation-promoting medium as described above in pDL-coated tissue culture flasks. Expanded cultures were then gently lifted from flasks: 10\% trypsin-EDTA solution (25300-054; Invitrogen) diluted in Earle's balanced salt solution for $6 \mathrm{~min}$ at $37^{\circ} \mathrm{C}$, and then an equal volume of $20 \%$ fetal calf serum (FCS) (10437-028; Invitrogen) in Dulbecco's PBS was added, and OPCs were rinsed off and pelleted at 220 relative centrifugal force for $15 \mathrm{~min}$. OPCs were resuspended in DMEM and pelleted again to rinse away all trypsin and FCS. A total of $2-3 \times 10^{6}$ aliquots of OPCs was resuspended in $100 \mu \mathrm{l}$ of Amaxa OPC nucleofection reagent (VPG-1009; Amaxa, Gaithersburg, MD) and 1.5$3.0 \mu \mathrm{g}$ of pC1-eGFP (CMV promoter-driven eGFP expression; 6084-1; Clontech, Mountain View, CA) plus the following: $2.5 \mu \mathrm{g}$ of pSPORT6UHRF1 (CMV promoter-driven mouse UHRF1 expression; MMM1013-64849; Open Biosystems, Huntsville, AL), 120 pmol of siControl nontargeting small interfering RNA (siRNA) pool (targets firefly luciferase; D-001206-13; Dharmacon, Lafayette, CO), 120 pmol of siGenome SMARTpool rat SOX10 (Dharmacon M-090803-00), 120 pmol of siGenome SMARTpool rat ZFP536 (Dharmacon M-082235-00). OPCplasmid/siRNA mixes were then electroporated with the Amaxa nucleofection apparatus, O-17 program. Transfected OPCs were plated at 30,000 cells/pDL-coated glass coverslips and incubated for $4 \mathrm{~d}$ in DMEM-Sato medium lacking PDGF and NT-3. Coverslips were then stained for GFP and either MBP or MOG expression as described above.

To assay effects of alterations of transcription factor expression on myelin gene expression, the percentage of transfected $\left(\mathrm{GFP}^{+}\right)$cells positive for early (MBP) or late (MOG) myelin gene expression was scored blind for each condition, 100-300 cells counted per coverslip, three coverslips scored per trial. Data were normalized to control levels of marker (MBP or MOG) expression within an individual trial (control for CMVUHRF1 plasmid transfections, CMV-eGFP plasmid only transfections; control for siZFP536 and siSOX10 pool transfections, nontargeting siRNA pool transfections). There were no significant differences in MBP or MOG expression between these two groups of controls (data not shown).

\section{Results \\ Generation of a comprehensive database of gene expression during OL differentiation}

To characterize the detailed temporal program of OL differentiation, we analyzed the global gene expression changes occurring in acutely isolated populations of primary cortical rat OPCs as they synchronously differentiated into mature OLs (see Materials and Methods). The purified $\mathrm{GalC}^{-} \mathrm{O}^{+}$OPCs were expanded in mitogens (PDGF and NT-3) in a defined, serum-free medium in the absence of thyroid hormone (T3) for $24 \mathrm{~h}$ before mRNA isolation to provide a baseline gene expression level in a highly enriched population of undifferentiated, $\mathrm{NG}^{+}$OPCs $(>90 \%$ OPCs, and $>95 \%$ OL-lineage cells) (Fig. $1 B$ ). Parallel cultures were then switched into differentiation-promoting medium, lacking PDGF/NT-3 and containing T3 (Barres et al., 1993, 1994), and mRNA was isolated at various time points as the OPCs differentiated into OLs over a $9 \mathrm{~d}$ period (Fig. $1 A$ ). After 3-4 d of culture, $90-95 \%$ of the cells had differentiated into multipolar $\mathrm{GalC}^{+}$and myelin basic protein ${ }^{+}$(MBP) OLs. Despite the absence of serum, $\sim 5-10 \%$ of the OPCs consistently differentiated into type 2 astrocytes (2As) instead of OLs (assayed by immunostaining) (data not shown), most likely because of the production of 2A-inducing signals by the differentiating OLs such as BMP-4 (see Table 2) (Mabie et al., 1997).

For the gene chip experiments, four independent OPC purifications and differentiation time courses were performed, providing four biological replicates per time point. Individual labeled mRNA samples were hybridized to the entire set of Affymetrix rat genomic U34 chips (A-C) to analyze gene expression on the total set of 26,202 rat gene and expressed sequence tag
(EST) probe sets available. Gene expression levels were assigned using the RMA method in GeneTraffic 3.2, and average gene expression level changes (relative to OPC levels) were calculated for each time point from the four biological replicates (see Materials and Methods). The results of the entire time course experiment are provided in supplemental Table S1 (available at www. jneurosci.org as supplemental material).

Because we were using an in vitro system to characterize OL differentiation, we also examined whether gene expression by the terminally differentiated OLs that we were generating in vitro mirrored that of OLs that are generated in vivo. We isolated acutely purified mature OLs from P10-P12 rat brains by immunopanning to $>99 \%$ purity (see Materials and Methods) and immediately purified their expressed RNA. Four independent acute OL purifications were performed and analyzed on Affymetrix rat genome U34A-C chips as described above, allowing us to compare gene expression in these acutely isolated OLs to the expression levels obtained in our in vitro time course (see below).

\section{Identification of a set of genes that are highly regulated during OL differentiation}

To identify genes likely involved in specifying the OL phenotype, we focused on the genes most strongly regulated during OL differentiation. Analysis of the gene expression data revealed that only 824 probe sets $(3.1 \%)$ demonstrated a more than fourfold change (up or down) relative to OPC levels at any time point or in the acutely purified OLs. To further characterize the gene expression data, we used a recently developed one-sample moderated Hotelling $\mathrm{T}^{2}$ statistic to rank the probe sets according to the statistical relevance of their expression changes over time (Tai and Speed, 2006). We compared the top 824 statistically ranked probe sets to the 824 most highly changing probe sets, and found that $695(84 \%)$ were indeed present on both lists. Interestingly, of the 129 probe sets identified solely by the statistical analysis, 61 (47\%) did change more than fourfold during OL differentiation: they were briefly induced and subsequently repressed, producing an overall change of more than fourfold over the analyzed course of OL differentiation, but never more than a fourfold change relative to OPC expression levels. In all, 953 strongly regulated probe sets were identified, which cumulatively corresponded to 778 unique genes (as assayed by determining which rat Unigene cluster each probe set sequence belongs to) (http://www.ncbi.nlm. nih.gov/) (supplemental Table S2, available at www.jneurosci.org as supplemental material). The expression time courses of these 953 selected probe sets are graphically depicted in Figure $1 C$. By comparing gene expression levels in OLs after 7 and $9 \mathrm{~d}$ of differentiation (days 7 and 9), we found that only 63 of these 953 probe sets change $>1.5$-fold and only 17 change $>2$-fold between these two time points, indicating that gene expression changes have mostly leveled off by the end of the time course. In comparison, we found that 244 of 953 probe sets change $>1.5$-fold and 83 of 953 probe sets change $>2$-fold between days 5 and 7 . Thus, as judged by the time course of gene expression changes, OL differentiation is unexpectedly prolonged, being nearly complete only by $7 \mathrm{~d}$ in vitro.

We next wanted to determine whether the OLs we had generated in our in vitro time course of OL differentiation were similar to OLs generated during normal development in vivo. To do this, we examined the degree of similarity between gene expression in the in vitro-generated OLs and the acutely isolated OLs. A total of 191 of the 953 probe sets showed a more than twofold difference between day 9 and acutely purified OL expression levels. Of these, 73 probe sets (representing 52 unique genes) were induced in 
vitro but expressed at levels similar to or lower than OPC levels in the acutely purified OLs, a pattern we would predict for genes specifically upregulated in the small percentage of type 2 astrocytes generated in our cultures that are not present among acutely purified OLs. Consistent with this, GFAP, which is expressed by 2As (Miller et al., 1985), and other well described astrocyte genes, shared this expression pattern. Therefore, these probable " $2 \mathrm{~A}$ " genes were excluded from additional analyses, leaving 880 probe sets (726 unique genes) identified as strongly regulated in differentiating OLs. Of the remaining 118 of 880 (13.4\%) probe sets that showed more than twofold differences between the in vitro and in vivo OLs, the majority demonstrated quantitative but not qualitative differences. For instance, 78 of these were induced in vitro, but were simply more strongly induced in the acutely purified OL samples ("up-acute") (Fig. 1D; supplemental Fig. S1C, available at www.jneurosci.org as supplemental material). As expected, among the OL genes that were induced in our in vitro time course were many previously characterized myelin-enriched genes (supplemental Table S3, available at www.jneurosci.org as supplemental material). In fact, we found no myelin genes expressed by the acutely purified OLs that were not also highly upregulated during OL differentiation in vitro, recapitulating previous findings that, unlike Schwann cells, most genes important to myelination are highly expressed by cultured OLs in the absence of neurons (Baumann and Pham-Dinh, 2001; Jessen and Mirsky, 2005). Remarkably, just seven probe sets were only induced in vivo while showing no change of expression in vitro ("acute") (Fig. 1C; supplemental Table S2, available at www. jneurosci.org as supplemental material). Thus, there was a remarkable similarity in gene expression between OLs generated in vitro and in vivo, with the exception primarily of a small group of genes that were likely derived from contaminating 2 As generated in vitro.

\section{Validation of gene chip data}

We next performed several analyses to confirm the accuracy of the OL differentiation-induced gene changes that we identified using gene profiling. First, we performed comparative RT-PCR to compare gene expression levels between acutely isolated OPCs and OLs. Using this approach, we assayed a total of 55 unique genes from our selected Affymetrix probe set list (supplemental Table S4, available at www.jneurosci.org as supplemental material). Although the selection of genes to be assayed was not random, it was based on gene ontology (mostly transcription factors and some additional putatively secreted proteins) rather than expression level or degree of induction/repression. The set of genes assayed included probe sets at all levels of expression (peak expression levels from 112 to 7274$)$, induction $(3.7-71 \times)$, and repression $(-3.9$ to $-18 \times)$. The set of assayed genes are therefore fairly representative of the distribution of expression patterns and levels seen in our 880 selected "non-2A pattern" probe sets.

Using this method, we were able to qualitatively detect and confirm not only robust gene expression changes, such as amyloid $\beta$ precursor-like protein 1 (APLP1) and apolipoproteinD $(A p o D)$, which were upregulated during OL differentiation by 10- and 71-fold, respectively, but also the 4-fold changes that served as the cutoff for our selected genes [such as transducin-like enhancer of split 1 (TLE1), which was upregulated by 4 -fold, and Ets variant gene 1 (ETV1), which was downregulated by 5 -fold] (Fig. 2A). Overall, we found that $93 \%$ of the gene expression changes assayed were independently verified (Fig. $2 B$; supplemental Table S4, available at www.jneurosci.org as supplemental material). The verification rate was similar for genes selected by our statistical method (96\%) and by identifying fourfold changes relative to OPC levels (91\%), with even genes selected only by fourfold changes (i.e., not statistically selected) having a high verification rate $(71 \%)$. These data therefore suggest that the majority of in vitro gene expression changes identified in our Affymetrix data set (excluding the $2 \mathrm{~A}$ genes) correspond to gene changes that occur during OL differentiation in vivo.

To determine whether any of the upregulated OL genes were normally expressed in white matter, we mined the Allen Brain Atlas, GENSAT, and BGEM in situ expression databases. Data were found for 70 of 84 highly expressed and upregulated OL genes identified from our time course, and nearly $80 \%$ of these ( 55 of 70 ) were expressed in white matter areas in one or more of the databases, including 33 that appeared to be most strongly expressed in white matter areas (Tables 1,2). These include several genes not previously cited as being expressed in OLs, such as genes coding for the transmembrane proteins GLTP and TMEM10, the secreted protein SEPP1, and a putative transcription factor CSRP1 (Allen Brain Atlas; http://www.brain-map. org/). The percentage of the genes expressed in white matter may be even higher than detected here. For instance, three of the genes not detected in white matter in the online databases analyzed (GPD1, DPYSL4, and BNIP3L) have in fact been previously cited as expressed in OLs (Tables 1, 2). In addition, the Allen Brain Atlas, on which the majority of these genes were found, displays only adult expression patterns. Given that some OL genes are downregulated by adulthood after a peak of expression during early myelination (for instance, SIRT2 expression peaking at P7; GENSAT database), and that 11 of the 15 genes not detected in white matter were assayed only on the Allen database, we expect that the actual percentage of our identified genes that are expressed by OLs at some stage in their development is likely to be $>80 \%$.

Finally, we examined whether we could independently verify the distinct temporal patterns of gene expression seen during OL differentiation at the protein level. For example, our data indicated that cyclic nucleotide phosphodiesterase 1 (CNP1) and myelin oligodendrocyte glycoprotein (MOG) were both induced in OLs, and that CNP1 was induced earlier than MOG (Fig. $3 A, B$ ). These findings are consistent with previous observations (Baumann and Pham-Dinh, 2001). To confirm these distinct temporal patterns of expression correspond to changes at the protein level, we immunostained purified OPCs as they differentiated into OLs at various time points (Fig. 2C). We found that the time courses of CNP1 and MOG protein expression closely paralleled the differential gene regulation seen in our gene chip data, indicating that the distinct phases of gene expression seen in the gene chip data can be recapitulated in normally differentiating OLs. Together, these lines of evidence based on the known literature, RT-PCR, in situ hybridization patterns, and protein levels confirm that the gene changes we identified are valid and significant.

\section{Terminal OL differentiation occurs in distinct temporal stages}

As shown in Figure 1, most of the 953 probe sets that are strongly up or downregulated as OLs differentiate do not show a temporally complex expression pattern, but are instead simply induced or repressed over the $9 \mathrm{~d}$ time course of OL differentiation. However, a closer examination of Figure $1 C$ shows that these broad classifications can be further subdivided by the initiation of expression level changes, with some genes altering their expression immediately on exposure to differentiating conditions, whereas 

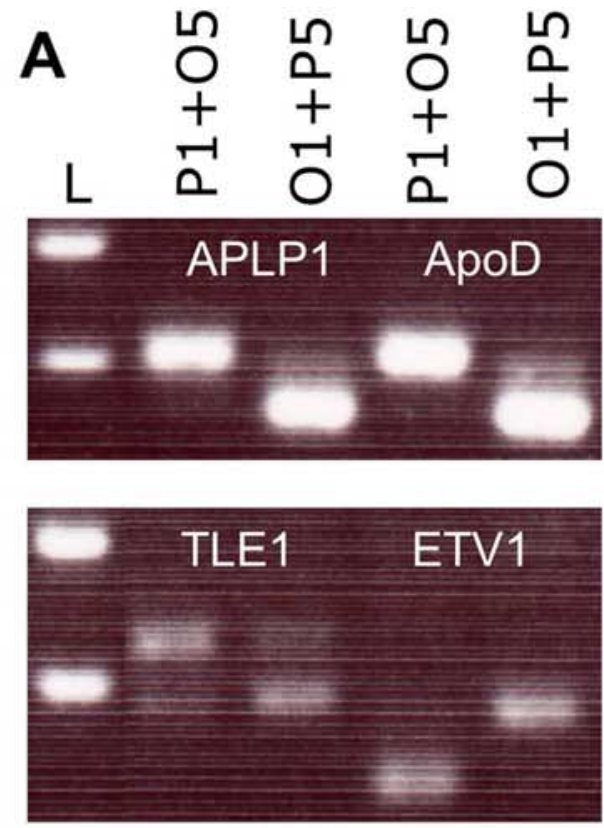

\section{B}
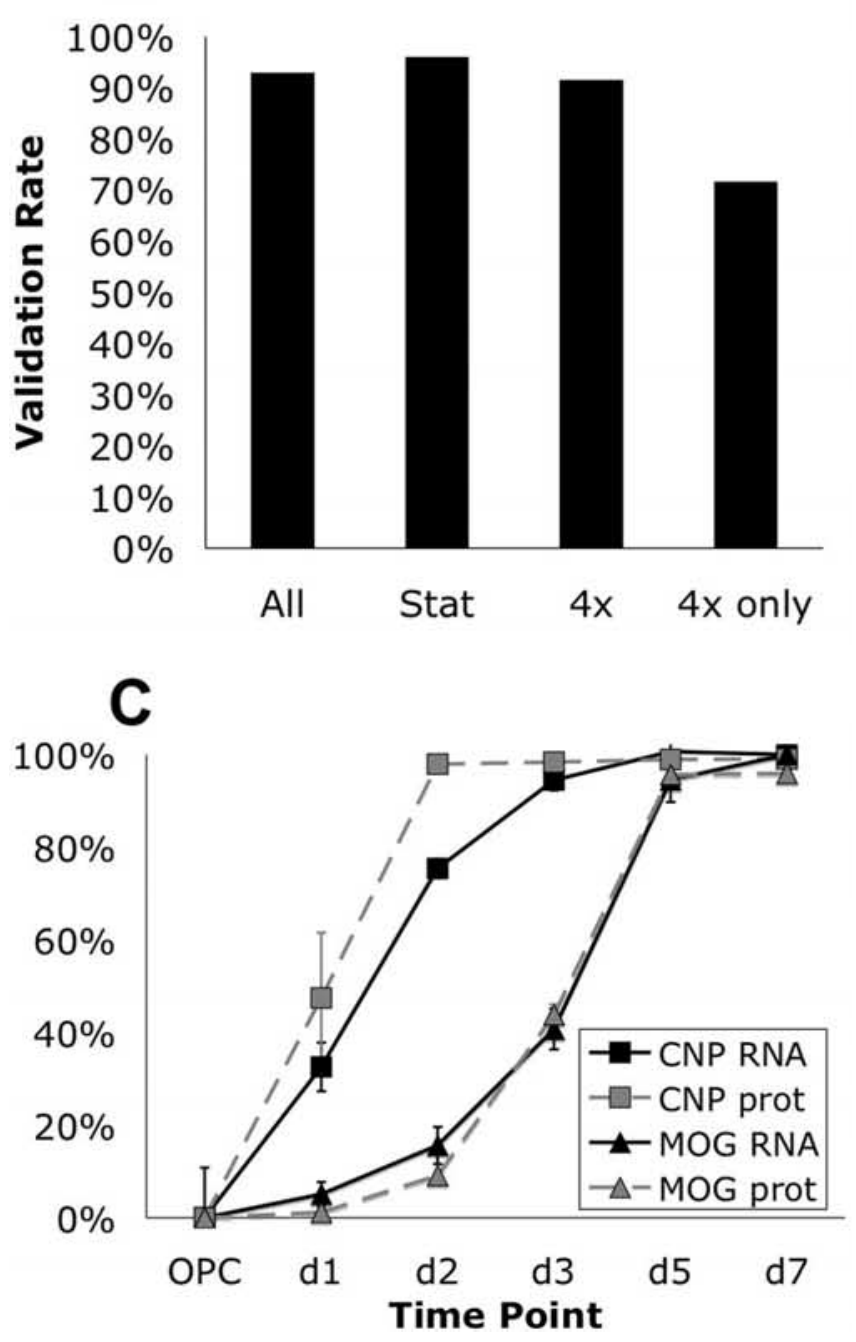

other genes are not induced or repressed until $24-48 \mathrm{~h}$ after the initiation of differentiation. The temporal profiles of gene expression changes detected in our OL differentiation time course were highly reproducible in replicate analyses (supplemental Fig. S1 $A-C$, available at www.jneurosci.org as supplemental material).

To investigate whether the transformation of OPCs into mature OLs represents a single terminal differentiation step or a series of sequential regulated stages, we plotted the levels of expression of several known myelin-enriched genes over the $9 \mathrm{~d}$ time course of OL differentiation. Interestingly, we found that these genes were not induced concurrently, but rather were segregated into two broad temporal categories: an early group of myelin genes that is induced immediately on differentiation (Fig. $3 A$ ) and a second group whose induction is delayed by $48 \mathrm{~h}$ or more (Fig. $3 B$ ). Similarly, we identified several other highly upregulated OL genes belonging to nearly every functional group that could be categorized as either early or late induced (supplemental Fig. S1 D-G and Table S5, available at www.jneurosci.org as supplemental material). For instance, the OL-enriched genes PNLIP and CHN2 are induced early during OL differentiation, whereas transferrin, SEPP1, and CTSL are all upregulated several days later (supplemental Table S5, available at www.jneurosci.org as supplemental material). Although we have divided these temporal phases into two main groups of genes (immediate induction vs $48-72 \mathrm{~h}$ delay), several of the OL genes, such as MAL and transferrin, were initially induced as late as $5 \mathrm{~d}$ after differentiation commenced, whereas other non-myelin-related genes, such as $I K B B$ and $C H E S 1$, were initially induced at intermediate time points ( 24 h delay).

The two broad phases of myelin gene induction are paralleled by similar changes in distinct sets of transcription factor genes, with one subset of transcription factors being upregulated rapidly early in OL differentiation (Fig. $4 \mathrm{~A}$ ), and a second subset of transcription factors that are induced only after a delay of 2 or more days (Fig. $4 B$ ). We also identified distinct groups of downregulated transcription factor genes that are either repressed early (Fig. 4C) or with a distinct delay (Fig. 4D) after the induction of OL differentiation. These downregulated transcription factors may represent genes that repress OL maturation; at least three of

$\leftarrow$

Figure 2. Verification of gene expression time courses. $A$, Verification of genomic expression data by comparative RT-PCR. The four examples shown are APLP1, ApoD, TLE1, and ETV1. The two comparative RT-PCRs for each gene are as follows: acute $\mathrm{OPC}-10 \mathrm{bp}$ tag plus acute $\mathrm{OL}-50$ bp tag (P1+05; 0L level upper band) and acute $0 \mathrm{~L}-10 \mathrm{bp}$ tag plus acute OPC $-50 \mathrm{bp}$ tag $(01+P 5 ; 0 \mathrm{~L}$ expression lower band); upregulated genes have stronger $0 \mathrm{~L}$ sample bands. L, 100 bp DNA ladder. $\boldsymbol{B}$, Cumulative comparative RT-PCR verification rates for tested genes. A gene was considered "verified" if its expression was qualitatively similar in both the gene chip and RT-PCR data (e.g., both showing higher levels in 0Ls relative to OPCs). A total of 51 of 55 selected Affymetrix probe sets was independently verified (all), including 37 of 40 tested transcription factor genes listed in supplemental Table $\$ 6$ (available at www.jneurosci.org as supplemental material); verification rates were 46 of 48 for probe sets identified by statistical analysis (stat) and 42 of 46 for probe sets changing $\geq 4 \times$ relative to OPC levels $(4 \times)$. Among probe sets selected only by fourfold changes (not statistically selected; $4 \times$ only), 5 of 7 tested probe sets were verified. C, Comparison of protein expression and gene expression time courses. Average CNP1 (L16532_at; black squares) and MOG (M99485_at; black triangles) RNA expression levels from Affymetrix at various time points during $0 \mathrm{~L}$ differentiation; expression levels relative to OPC levels expressed on a $\log _{2}$ scale, shown as percentages of maximal day 7 expression levels. To assay protein expression, purified P7 OPCs were cultured as depicted in Figure $1 \mathrm{~A}$. The percentages of healthy cells strongly expressing CNP1 (gray squares) and MOG (gray triangles) were assayed by immunostaining at the time points indicated. All data points are presented $\pm \mathrm{SEM}(n=4$ for RNA; $n=3$ for protein). 
Table 1. Top 50 upregulated genes during $0 \mathrm{~L}$ differentiation

\begin{tabular}{|c|c|c|c|c|c|c|c|}
\hline Probe set ID & Fold change & Peak level & Gene name & Gene symbol & Allen & $B / G$ & Prev cit \\
\hline D28111_g_at & 119.43 & 5316 & Myelin-assoc OL basic protein & MOBP & Yes* & - & $1^{*}$ \\
\hline K00512_at & 98.36 & 14,359 & Myelin basic protein & $M B P$ & Yes* & Yes* & $1^{*}$ \\
\hline M99485_at & 97.68 & 3636 & Myelin oligodendrocyte glycoprotein & MOG & Yes* & - & $1^{*}$ \\
\hline rc_Al233181_at & 93.05 & 2404 & ESTs, no homologies found & & - & - & - \\
\hline rC_Al072770_s_at & 77.71 & 6127 & Proteolipid protein & PLP & Yes* & Yes* & $1^{*}$ \\
\hline X55572_at & 71.01 & 5230 & Apolipoprotein D & $A P O D$ & Yes* & Yes* & $2^{*}$ \\
\hline rC_AA891719_at & 66.72 & 3448 & Ectonuc. pyrophos./phosphodiesterase 6 & ENPP6 & Yes $^{* \wedge}$ & - & 3 \\
\hline D88534_s_at & 60.55 & 6354 & Pancreatic lipase & PNLIP & No & - & 4 \\
\hline rc_AA901342_at & 49.87 & 7334 & Claudin 11/0L specific protein & OSP & - & - & $1^{*}$ \\
\hline D38380_g_at & 44.63 & 4822 & Transferrin & Tf & No & Yes* & $5^{*}$ \\
\hline rc_Al101500_at & 38.59 & 4165 & ESTs, no homologies found & & - & - & - \\
\hline M22357_g_at & 38.32 & 6043 & Myelin-associated glycoprotein & MAG & Yes* & Yes* & $1^{*}$ \\
\hline rC_AA893670_at & 36.00 & 1406 & Tumor protein D52 & TPD52 & No & - & - \\
\hline rc_Al009946_at & 31.34 & 1129 & Carboxypeptidase M & $C P M$ & Yes & - & $6^{*}$ \\
\hline rc_Al236200_at & 29.86 & 882 & Mannosidase 1, $\alpha$ & MAN1A1 & Yes & - & - \\
\hline rc_AA800851_s_at & 29.04 & 7016 & Septin 5 & SEPT5 & No & No & 7 \\
\hline rC_Al228110_s_at & 26.91 & 7707 & UDP-glucuronosyltransferase 8 & UGT8 & Yes* & - & $1^{*}$ \\
\hline rC_Al232970_at & 25.81 & 1352 & Glycerol 3-phosphate dehydrogenase & GPD1 & No & - & $8^{*}$ \\
\hline rC_AI232373_at & 25.63 & 2305 & Thyroid hormone-response protein-1 & $A P X L$ & No & - & 9 \\
\hline S85184_at & 24.93 & 2314 & Cathepsin L & CTSL & Yes & Yes $^{* \wedge}$ & 10 \\
\hline rc_Al144614_at & 23.75 & 709 & Transmembrane protein TMP10 & TMEM10 & Yes $^{* \wedge}$ & - & 11 \\
\hline rC_Al230247_s_at & 22.32 & 606 & Selenoprotein $P$ & SEPP1 & Yes $^{* \wedge}$ & Yes & 12 \\
\hline rc_Al058796_at & 21.71 & 4441 & Hypothetical protein DKFZp566N034 & & - & - & - \\
\hline rC_Al232194_at & 21.41 & 1429 & Chimerin 2 & CHN2 & Yes & Yes & 13 \\
\hline D28560_at & 21.11 & 3809 & Ectonuc. pyrophos./phosphodiesterase 2 & ENPP2 & Yes & Yes* & $14^{*}$ \\
\hline rC_Al072835_at & 20.82 & 757 & Semaphorin $5 A$ & SEMA5A & No & Yes & $15^{*}$ \\
\hline rC_AA943765_at & 19.84 & 2000 & Synaptotagmin-like 2 & SYTL2 & Yes & - & 16 \\
\hline rC_Al072720_at & 19.70 & 613 & $\mathrm{~N}$-acetylgalactosaminyltransferase 5 & GALNT5 & No & No & - \\
\hline rC_AA925520_at & 19.70 & 1031 & ADP-ribosylation guan. nuc. factor $6 a-$-like & & No & - & - \\
\hline rC_Al007768_at & 19.56 & 1059 & Protein phosphatase 1 , reg. subunit $14 a$ & PPP1R14A & Yes $^{* \wedge}$ & - & 17 \\
\hline rC_AI234146_at & 19.03 & 3529 & Cysteine and glycine-rich protein 1 & CSRP1 & Yes $^{* \wedge}$ & - & 18 \\
\hline rC_Al112149_at & 18.77 & 2106 & Endothelial diff., LPA G-prot.-coup.-R, 2 & EDG2 & Yes & Yes* & $19^{*}$ \\
\hline rC_Al229178_at & 18.25 & 1324 & Fibroblast growth factor receptor 2 & FGFR2 & Yes* & Yes & $20^{*}$ \\
\hline AF016269_at & 18.13 & 1679 & Kallikrein 6 & KLK6 & Yes* & - & $21^{*}$ \\
\hline rc_Al233688_at & 18.00 & 430 & Plastin 1 & PLS1 & - & - & - \\
\hline rC_AA963260_at & 17.75 & 4838 & Erythrocyte memb. prot. band 4.1-like 2 & EPB41L2 & Yes & $Y_{e s}^{* \wedge}$ & 22 \\
\hline rc_Al013705_at & 17.03 & 1260 & Protease, serine, 11 (IGFbinding) & HTRA1 & No & - & 23 \\
\hline rc_Al071861_at & 17.03 & 741 & Bone morphogenetic protein 4 & BMP4 & - & Yes & $24^{*}$ \\
\hline X68101_at & 16.91 & 2114 & Dedicator of cytokinesis 9 & DOCK9 & Yes & - & 25 \\
\hline rC_Al232059_at & 16.91 & 334 & Aspartoacylase & ASPA & Yes & Yes* $^{*}$ & $1^{*}$ \\
\hline rC_AA899764_at & 16.91 & 2811 & Tetraspanin 2 & TSPAN2 & Yes* & - & $26^{*}$ \\
\hline rC_Al013502_at & 16.91 & 2344 & Lipoma HMGIC fusion partner-like 2 & LHFPL2 & No & - & - \\
\hline rC_AA850738_at & 16.68 & 1515 & HCV NS3-transactivated protein 2 & GRAMD3 & Yes $^{* \wedge}$ & - & - \\
\hline rc_Al029544_at & 16.00 & 362 & Spermatogenesis assoc. glu-rich prot. $4 f$ & SPEER4F & - & - & - \\
\hline rC_Al030921_i_at & 15.78 & 5321 & Chemokine-like factor super family 7 & CMTM7 & No & - & - \\
\hline rC_Al112086_at & 15.56 & 1395 & Prot. tyrosine phos'ase, receptor type, D & PTPRD & Yes & - & 27 \\
\hline AJ131902_g_at & 15.45 & 2832 & Growth arrest specific 7 & GAS7 & Yes & - & 28 \\
\hline rc_Al009484_at & 15.45 & 3008 & Gelsolin & GSN & Yes* $^{*}$ & Yes* $^{*}$ & $29^{*}$ \\
\hline rc_AA945734_at & 15.35 & 863 & Putative phosphatase subunit & & No & - & - \\
\hline U37142_at & 15.14 & 6444 & Brevican & $B C A N$ & Yes & - & $30^{*}$ \\
\hline
\end{tabular}

The 50 unique genes most highly induced during $0 \mathrm{~L}$ differentiation are listed. Genes listed in bold are present in both Tables 1 and 2 . In cases in which more than one Affymetrix probe set (Probe set ID) corresponding to the same gene was highly regulated, only the most strongly regulated is listed. Note that genes with a likely $2 \mathrm{~A}$ expression pattern are not included. Fold change, Highest expression level/lowest expression level in time course; positive numbers are upregulated relative to OPCS. Peak level, Highest raw level of expression seen at any time point in the gene chip experiment. Allen, B/G, Determination whether in situ expression of listed genes is detected in white matter areas of the CNS as determined from the Allen Brain Atlas (http://www.brain-map.org/) or the BGEM/GENSAT databases (http://www.stjudebgem.org/, http://www.ncbi.nlm.nih.gov/). - Not found. 'White matter enriched expression patterns not previously reported in the literature. Data from the BGEM and GENSAT databases were identical except for EPBL41L2 (only found on GENSAT), If and SEPT5 (only found on BGEM). Prev cit, If expression was previously reported in the CNS, the reference is listed (see supplemental material, available at www.jneurosci.org). - , Not previously reported in CNS. In Allen, B/G, and Prev cit, the asterisk $\left(^{*}\right)$ indicates a white matter enriched expression pattern or previously reported expression specifically in CNS white matter or OLs.

these genes, early growth response 1 (EGR1), myelin transcription factor 1 (MYT1), and myelocytomatosis oncogene (MYC), have been previously implicated in repressing OL differentiation (Sock et al., 1997; Orian et al., 2001; Nielsen et al., 2004). Interestingly, a few transcription factors are expressed with a pattern similar to the late-stage cell cycle control genes (Figs. 3D, 4E), and two of these, UHRF1 and HMGB2, have been previously implicated in cell cycle progression (Yamazaki et al., 1995; Arima et al., 2004).
These data provide evidence that OL differentiation is an unexpectedly prolonged multistep process.

Distinct stages of OL differentiation can be independently regulated

Do the temporally distinct waves of gene expression we observe represent discrete stages of normal OL differentiation? To begin to address this question, we tested whether the early and later 
Table 2. Top 50 0L-specific expressed genes

\begin{tabular}{|c|c|c|c|c|c|c|c|}
\hline Probe set ID & OL level & Fold change & Gene name & Gene symbol & Allen & $\mathrm{B} / \mathrm{G}$ & Prev cit \\
\hline M58369_at & 18,758 & 48.84 & Pancreatic lipase & PNLIP & No & - & 4 \\
\hline K00512_at & 14,359 & 98.36 & Myelin basic protein & $M B P$ & Yes $^{*}$ & Yes* & $1^{*}$ \\
\hline rC_Al070277_s_at & 13,729 & 31.78 & Proteolipid protein & $P L P$ & Yes $^{*}$ & Yes* $^{*}$ & $1^{*}$ \\
\hline X776489cds_g_at & 12,541 & 9.13 & CD9 antigen (p24) & $\operatorname{CD} 9$ & Yes* & - & $31^{*}$ \\
\hline rC_AA858590_at & 10,833 & 12.47 & ESTS, no homologies found & & - & - & - \\
\hline rc_AA799614_at & 10,533 & 10.85 & Sirtuin 2 & SIRT2 & - & Yes*^ $^{* \wedge}$ & 32 \\
\hline M81225_at & 10,485 & 5.06 & Farnesyltransferase, (AAX box, $\alpha$ & FNTA & Yes & - & 33 \\
\hline rC_AA848813_at & 9064 & 4.86 & Deleted in polyposis 1 & REEP5 & Yes & - & - \\
\hline L16532_at & 9059 & 12.64 & 2', 3'-Cyclic nuc. 3'-phosphodiesterase & CNP1 & Yes $^{*}$ & - & $1^{*}$ \\
\hline rC_AI233362_at & 8588 & 4.47 & Lysosomal associated membrane protein 1 & LAMP1 & Yes & - & 34 \\
\hline rC_Al228110_S_at & 7707 & 26.91 & UDP-glucuronosyltransferase 8 & UGT8 & Yes $^{*}$ & - & $1^{*}$ \\
\hline rc_AA901342_at & 7334 & 49.87 & Claudin 11/0L specific protein & OSP & - & - & $1^{*}$ \\
\hline rc_AA943617_at & 7274 & 9.58 & Amyloid $\beta$ precursor-like protein 1 & APLP1 & Yes & No & $35^{*}$ \\
\hline rC_Al176595_s_at & 7174 & 16.11 & Cathepsin L & CTSL & Yes & Yes** $^{* \wedge}$ & 10 \\
\hline rC_AA800851_s_at & 7016 & 29.04 & Septin 5 & SEPT5 & No & No & 7 \\
\hline rC_AA900632_s_at & 6896 & 5.31 & Glycolipid transfer protein & GLTP & Yes*^ $^{* \wedge}$ & - & 36 \\
\hline rC_Al007851_at & 6808 & 19.29 & Hypothetical protein DKFZp566N034 & & - & - & - \\
\hline U37142_at & 6444 & 15.14 & Brevican & BCAN & Yes & - & $30^{*}$ \\
\hline rC_Al103957_at & 6141 & 4.08 & CD81 antigen & CD81 & Yes* & - & $37^{*}$ \\
\hline M22357_g_at & 6043 & 38.32 & Myelin-associated glycoprotein & MAG & Yes $^{*}$ & Yes$^{*}$ & $1^{*}$ \\
\hline J03588_at & 5945 & 10.78 & Guanidinoacetate methyltransferase & GAMT & Yes $^{*}$ & - & $38^{*}$ \\
\hline rC_Al102031_at & 5859 & 3.86 & Bridging integrator 1 & BIN1 & Yes & No & 39 \\
\hline rC_AA997651_at & 5842 & 4.17 & Phytanoyl-CoA hydroxylase interacting prot. & PHYHIP & No & - & 40 \\
\hline M31174_at & 5828 & 4.38 & Thyroid hormone receptor $\alpha$ & THRA & Yes & No & $41^{*}$ \\
\hline D50093_s_at & 5638 & 6.19 & Prion protein (p27-30) & PRNP & Yes & No & 42 \\
\hline AF009604_at & 5574 & 4.86 & $\mathrm{SH} 3$ domain protein $2 \mathrm{C} 1$ & SH3D2C1 & - & - & 43 \\
\hline rC_Al030921_i_at & 5321 & 15.78 & Chemokine-like factor superfamily 7 & CMTM7 & No & - & - \\
\hline rC_AA800549_at & 5317 & 5.70 & Prot. phosphatase 1, reg. subunit 16B & PPP1R16B & Yes & Yes*^ $^{* *}$ & 44 \\
\hline rc_AA875232_at & 5316 & 6.11 & Myelin protein zero-like 1 & MPZL1 & Yes*^ $^{* \wedge}$ & - & 45 \\
\hline D28111_g_at & 5316 & 119.43 & Myelin-assoc OL basic protein & MOBP & Yes* & - & $1^{*}$ \\
\hline X55572_at & 5230 & 71.01 & Apolipoprotein D & $A P O D$ & Yes $^{*}$ & Yes* $^{*}$ & $2^{*}$ \\
\hline rC_AA997237_at & 5087 & 6.41 & ESTS, no homologies found & & - & - & - \\
\hline U28938_at & 4956 & 9.65 & Prot. Y-phosphatase, receptor type, 0 & PTPRO & No & - & 46 \\
\hline rC_Al103774_at & 4944 & 8.46 & Dynein light chain-2 & DLC2 & Yes & - & 47 \\
\hline rc_AA963260_at & 4838 & 17.75 & Erythrocyte memb. prot. band 4.1-like 2 & EPB41L2 & Yes & Yes** $^{* \wedge}$ & 22 \\
\hline D38380_g_at & 4822 & 44.63 & Transferrin & If & No & Yes $^{*}$ & $5^{*}$ \\
\hline U52103_at & 4792 & 10.06 & Dihydropyrimidinase-like 4 & DPYSL4 & No & - & $48^{*}$ \\
\hline rC_Al070110_at & 4782 & 7.26 & C1q-related factor precursor & C1QL1 & - & - & 49 \\
\hline AF034237_s_at & 4765 & 5.74 & Pleckstrin homology domain-containing A-1 & PLEKHA1 & Yes & - & 50 \\
\hline rC_AA892496_at & 4642 & 16.80 & Chimerin 2 & CHN2 & Yes & Yes & 13 \\
\hline rC_AA892919_g_at & 4605 & 3.71 & ESTS, no homologies found & & - & - & - \\
\hline rC_AA901317_at & 4484 & 14.22 & ESTS, no homologies found & & - & - & - \\
\hline X07648cds_g_at & 4463 & 5.10 & Amyloid $\beta$ precursor protein & $A P P$ & - & - & $51^{*}$ \\
\hline rC_AA925083_at & 4418 & 7.78 & Striatin, calmodulin binding protein & STRN & Yes & Yes & 52 \\
\hline rC_Al013011_at & 4284 & 4.53 & Guanine nucleotide binding prot., a12 & GNA12 & No & Yes** $^{* \wedge}$ & 53 \\
\hline rC_Al011991_at & 4266 & 6.19 & Ras homolog gene family, member $G$ & RHOG & Yes $^{*}$ & No & $54^{*}$ \\
\hline rC_AA859938_at & 4254 & 5.21 & BCL2/adenovirus E1B 19 kDa-interac. prot. 3 & BNIP3L & No & No & $55^{*}$ \\
\hline rC_AA998207_at & 4149 & 4.47 & Junction cell adhesion molecule 3 & JAM3 & - & - & 56 \\
\hline AF026529_s_at & 4076 & 5.62 & Stathmin-like 4 & STMN4 & Yes & - & $57^{*}$ \\
\hline rC_AA926231_at & 4011 & 8.06 & EGF receptor pathway substrate 15 & EPS15 & Yes & - & 58 \\
\hline
\end{tabular}

Top $50 \mathrm{OL}$-specific expressed genes. The 50 unique genes most strongly expressed specifically in mature $0 \mathrm{Ls}$ are listed. Genes listed in bold are present in both Tables 1 and 2 . In cases in which more than one Affymetrix probe set (Probe set ID) corresponding to the same gene was highly expressed, only the most strongly expressed is listed. Genes with a likely $2 \mathrm{~A}$ expression pattern were excluded. $0 \mathrm{~L}$ level, Highest raw expression level seen at the day 7, day 9, or acutely purified $\mathrm{OL}$ time points (mature $0 \mathrm{Ls}$ ). Fold change, Highest expression level/lowest expression level in time course, positive numbers are upregulated relative to $\mathrm{OPCs}$. Allen, $\mathrm{B} / \mathrm{G}$, Determination whether in situ expression of listed genes is detected in white matter areas of the CNS as determined from the Allen Brain Atlas (http://www.brain-map.org/) or the BGEM/GENSAT databases (http://www.stjudebgem.org/, http://www.ncbi.nlm.nih.gov/); - , Not found. "White matter enriched expression patterns not previously reported in the literature. Data from the BGEM and GENSAT databases were identical except for EPBL47L2 (only found on GENSAT), If and SEPT5 (only found on BGEM). Prev cit, If expression was previously reported in the CNS, the reference is listed (see supplemental material, available at www.jneurosci.org). —, Not previously reported in (NS. In Allen, B/G, and Prev cit, the asterisk (*) indicates a white matter enriched expression pattern or previously reported expression specifically in CNS white matter or 0Ls. Note that AF030089UTR\#1_at (ANIA4, 5345 at day 9) and AF023087_s_at (EGR1, 4290 at day 9) were excluded because they are more highly expressed in OPCs (Table 4).

phases of OL gene expression could be independently regulated. We compared the effects of disrupting the normal expression of a transcription factor known to be involved in regulating the initial stages of OL differentiation, SOX10, to the effects of disrupting transcription factors regulated during the later phase of OL differentiation. We chose ZFP536, a putative transcription factor that is upregulated eightfold late in OL differentiation (Fig. $4 B$; supplemental Table S6, available at www.jneurosci.org as supplemental material), and UHRF1, discussed above, which is repressed eightfold late in OL differentiation (Fig. 4E; supplemental Table S6, available at www.jneurosci.org as supplemental material). These genes are two of the transcription factors that are most robustly regulated during the later phase of terminal OL differentiation. Comparatively, SOX10 is induced 2.7 -fold, be- 


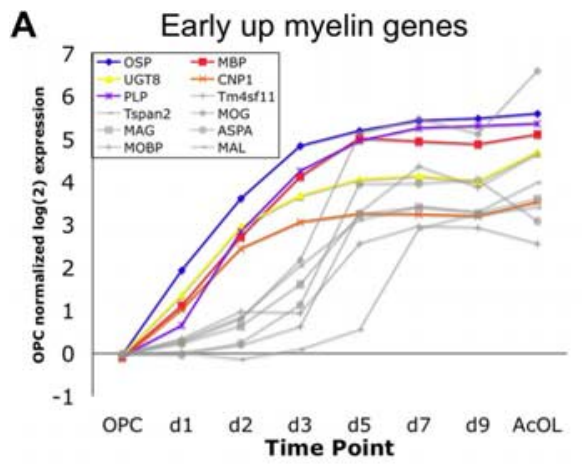

\section{Down cell cycle / DNA genes}

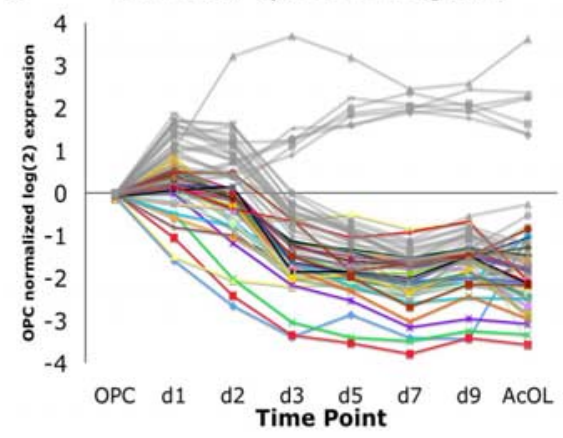

E

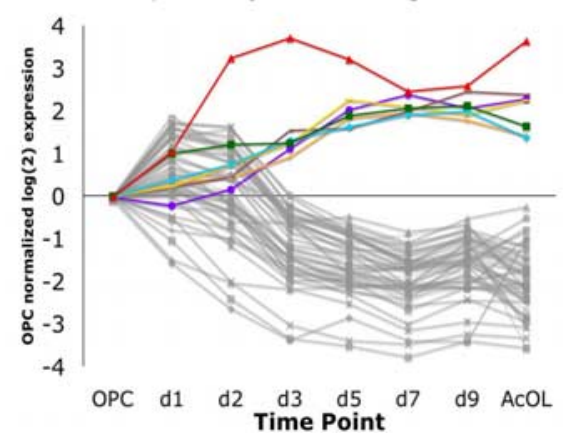

Figure 3. Temporal expression patterns of regulated myelin and cell cycle control/DNA replication genes. $\boldsymbol{A}, \boldsymbol{B}$, Myelinenriched genes are induced in two distinct phases during $0 \mathrm{~L}$ differentiation (supplemental Table S3, available at www.jneurosci. org as supplemental material). $\boldsymbol{A}$, Genes induced immediately after PDGF/NT3 withdrawal and T3 exposure are highlighted in color (OSP, MBP, UGT8, CNP1, PLP), with late-induced myelin-related genes shown in gray. $\boldsymbol{B}$, Genes whose induction is delayed after PDGF/NT3 withdrawal and T3 exposure are highlighted in color (Tm4sf11, Tspan2, MOG, MAG, ASPA, MOBP, MAL). C-E, Expression patterns of all regulated cell cycle/DNA replication genes (supplemental Table S7, available at www.jneurosci.org as supplemental material). C, Genes repressed soon after PDGF/NT3 withdrawal and T3 exposure are highlighted in color, with remaining genes shown in gray. $\boldsymbol{D}$, Genes that immediately peak and are subsequently downregulated after PDGF/NT3 withdrawal and T3 exposure are highlighted in color. $\boldsymbol{E}$, Genes induced after PDGF/NT3 withdrawal and T3 exposure are highlighted in color. In all graphs, expression levels are determined as average fold changes relative to average OPC levels and expressed on a $\log _{2}$ scale at the time points indicated. AcOL, Acutely purified $0 \mathrm{~L}$ sample.

ginning immediately on induction of OL differentiation [supplemental Table S1, probe set AJ001029_at (available at www. jneurosci.org as supplemental material)].

To disrupt the normal expression patterns of these genes, we either overexpressed UHRF1 to prevent its normal repression, or knocked down ZFP536 or SOX10 to prevent their normal induction during OL differentiation. Purified P7 OPCs were cultured and then transfected with either CMV promoter-driven mouse UHRF1, or with siRNA pools designed to knock down ZFP536 or SOX10 expression. Transfected cells were then cultured in differentiation-promoting medium for $4 \mathrm{~d}$ and stained for either early-induced $(M B P)$ or later-induced $(M O G)$ myelin gene ex- pression (Fig. 5). Knock down of SOX10 and ZFP536 by siRNA transfection was confirmed by semiquantitative RT-PCR (supplemental Fig. S3, available at www. jneurosci.org as supplemental material). As expected, knock down of SOX10 levels reduced the number of MBP-expressing cells relative to control, nontargeting siRNA transfections (Fig. 5A,B). Comparably, knock down of late-induced ZFP536 or overexpression of late-repressed UHRF1 reduced the number of MOGexpressing cells relative to controls (Fig. $5 C-E)$. A more thorough examination of the effects of disrupting early and late transcription factor expression is shown in Figure $5 F$. We find that preventing normal regulation of the late-phase transcription factors, by blocking the normal induction of ZFP536 or the normal repression of UHRF1, selectively reduces expression of the late-phase gene $M O G$ without disrupting normal levels of $M B P$ expression. Conversely, reduction of normal levels of SOX10, which is induced early and has been previously shown to be involved in early OL generation (Stolt et al., 2002), selectively blocks $M B P$ expression without affecting levels of the later-induced gene $M O G$. The fact that we do not observe $100 \%$ repression of $M B P$ or $M O G$ in these experiments likely reflects a combination of incomplete knock down (in the case of siRNA transfections) coupled with the presence of compensatory (potentially similarly regulated) transcription factors in the OLs. Cumulatively, these results provide evidence that the two discrete phases of myelin gene expression that we observe (Fig. $3 A, B$ ) are regulated by distinct programs.

\section{Functional classification of genes regulated during OL differentiation}

To obtain a more comprehensive appreciation of the gene expression changes occurring during OL differentiation, we grouped all of the regulated genes we identified by biological function (Fig. 6). One hundred seventy-six of these genes were either ESTs or genes with no known biological function, many of which may represent previously unrecognized genes important to OL differentiation. Among known genes, those involved in signal transduction and metabolism make up the largest classes of regulated genes. The several identified regulated genes involved in either cytoskeleton/motility or membrane trafficking/metabolism may be involved in either myelin sheath generation (induced genes) or OPC migration (repressed genes). In addition, the 51 transcription factors identified as dynamically regulated during OL differentiation could serve as intrinsic regulators of OL myelin gene expression (supplemental Table S6, available at www.jneurosci.org as supplemental material). Differential expression of the majority of these transcription 
factor genes was independently verified by comparative RT-PCR (supplemental Table S4, available at www.jneurosci.org as supplemental material).

An important area of investigation has been understanding how OPC proliferation is coupled to OL differentiation, and the nature of the intracellular timer within OPCs that counts and limits the maximum number of times they can divide before differentiating. To obtain a more detailed view of cell cycle gene regulation, we plotted the time courses of the expression of all the regulated cell cycle and DNA repair/ replication genes we identified (Fig. $3 C-$ $E$ ). As indicated in Figure $6 B$, most of these genes were either immediately suppressed as expected (Fig. 3C), or briefly peaked before being repressed (Fig. 3D). Predictably, regulated genes associated with the early $\mathrm{G}_{1} / \mathrm{S}$ and $\mathrm{S}$ phases of mitosis are repressed early (Fig. 3C; supplemental Table S7, available at www.jneurosci.org as supplemental material). Genes associated with the later phases of mitosis underwent a brief $2 \mathrm{~d}$ boost in expression level followed by repression, as OPCs already committed to DNA synthesis completed the cell cycle after being switched into differentiationpromoting medium (Fig. 3D; supplemental Table S7, available at www.jneurosci. org as supplemental material). These include a large number of genes involved in regulating the $\mathrm{G}_{2} / \mathrm{M}$ phase transition or progression through $\mathrm{M}$ phase. Finally, we identified a few cell cycle associated genes that are highly induced during OL differentiation, such as CHES1, PKMYT1, and CDKN1C (Fig. 3E; supplemental Table S7, available at www.jneurosci.org as supplemental material), which are, consistent with their expression patterns, known inhibitors of the cell cycle (Lee et al., 1995; Booher et al., 1997; Pati et al., 1997).

\section{Analysis of genes most highly expressed by OLs or induced during differentiation}

To learn more about OL differentiation and function, we next generated two gene lists: first, a list of the 50 genes most strongly induced during OL differentiation (Table 1); second, a list of the 50 genes that were most highly expressed by mature OLs but not by OPCs (Table 2). To generate this second list, we chose the 50 genes with the highest expression levels from among the day 7 , day 9, or acute OL data points within our selected list of highly regulated genes (supplemental Table S2, available at www. jneurosci.org as supplemental material), excluding those genes with higher expression at the earlier time points, or genes with likely $2 \mathrm{~A}$ expression patterns. This allowed us to focus on genes specifically enriched in the mature OLs. Only 16 genes are present on both lists, whereas the remaining 68 genes are only present on one of the two lists. Interestingly, expression of only 32 of these 84 genes had previously been described in OLs. Among the remain-
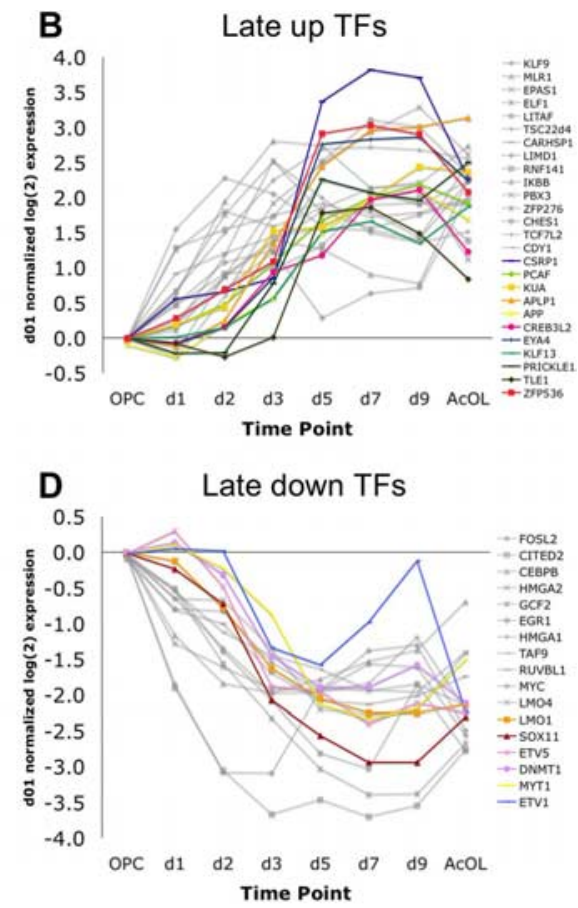

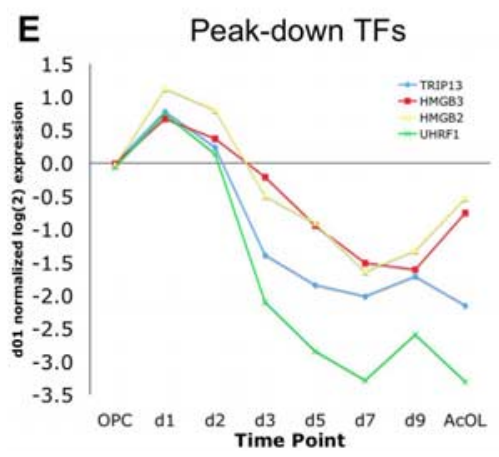

Figure 4. Temporal expression patterns of regulated transcription factor genes. $\boldsymbol{A}, \boldsymbol{B}$, Transcription factor genes induced during OL differentiation (supplemental Table 56 , available at www.jneurosci.org as supplemental material). $\boldsymbol{A}$, Genes induced imme作

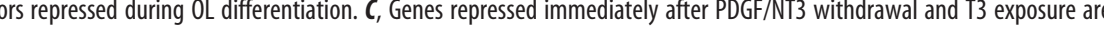
PDGF/NT3 withdrawal and T3 exposure are highlighted in color (DAT1, SOX11, ETV5, DNMT1, MYT1, ETV1). E, Transcription factors that immediately peak and are later repressed after PDGF/NT3 withdrawal and T3 exposure (TRIP13, HMGB2, HMGB3, UHRF1) are plotted. In all graphs, expression levels are determined as average fold changes relative to average OPC levels and expressed on a $\log _{2}$ scale at the time points indicated. AcOL, Acutely purified OL sample; TF, transcription factor.

ing 52 genes, 34 had been detected generally in the brain or only in neurons, and for 18 (including 7 uncharacterized ESTs) no CNS expression had been previously reported. In addition, 13 of these 52 genes are most highly expressed in white matter areas of the CNS as shown by in situ hybridization (Tables 1,2). We have therefore identified several genes with strong OL expression patterns, which have been previously unreported. Although a complete discussion of all of these genes is beyond the scope of this paper, we discuss some of the most interesting genes that we identified below.

We first examined genes that are both highly induced and highly expressed by OLs. As expected, 6 of the 16 genes present on both of these gene lists are known myelin genes: myelin basic protein $(M B P)$, proteolipid protein $(P L P), U D P$ glycosyltransferase 8 (UGT8), claudin-11 (also called OSP), myelin associated glyco- 

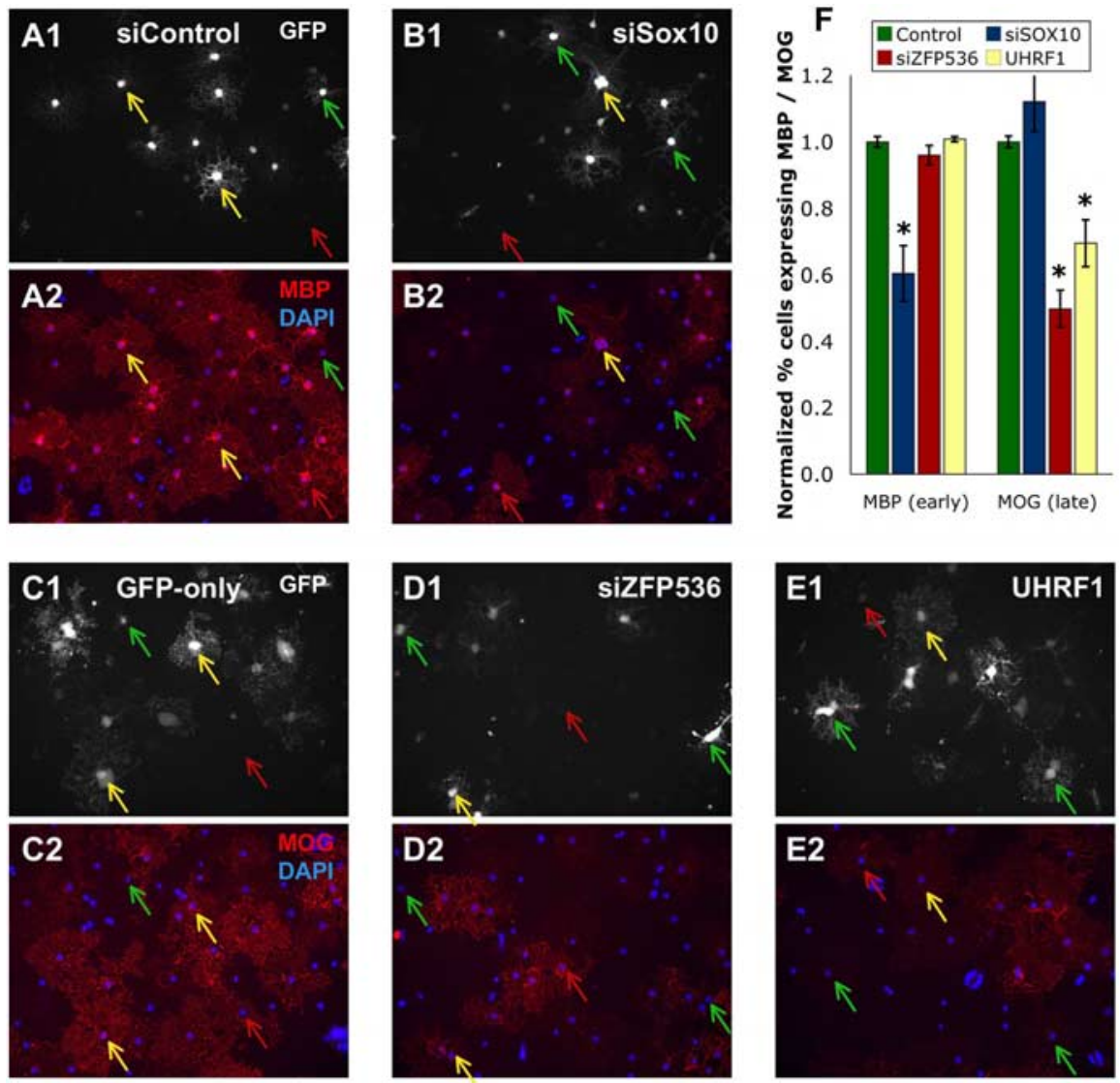

Figure 5. Distinct stages of $0 \mathrm{~L}$ differentiation are differentially regulated. $\boldsymbol{A}-\boldsymbol{E}$, Cultured $\mathrm{OPC}$ were transfected with a CMVeGFP expression vector alone $(\boldsymbol{C})$, or with either a pool of nontargeting siRNAs $(\boldsymbol{A})$, siRNAs targeting rat $\mathrm{SOX10}(\boldsymbol{B})$, siRNAs targeting rat ZFP536 (D), or a CMV-UHRF1 overexpression plasmid (E). Transfected OPCs were then cultured in medium lacking OPC mitogens (no PDGF or NT-3) to promote differentiation for $4 \mathrm{~d}$ in vitro, after which cells were costained for GFP expression to mark transfected cells (A1-E1) and either MBP (A2-B2) or MOG (C2-E2) expression to assay the levels of early (MBP) or late (MOG) induced myelin gene expression. The yellow arrows denote transfected cells (GFP ${ }^{+}$) expressing indicated markers (MBP or MOG), the green arrows indicate transfected cells not expressing indicated markers, and the red arrows denote untransfected cells $\left(\mathrm{GFP}^{-}\right)$expressing indicated markers. DAPI, $4^{\prime}, 6^{\prime}$-Diamidino-2-phenylindole. $\boldsymbol{F}$, Effects of individual transcription factor knockdown or overexpression on MBP and MOG expression in transfected OLs; control, CMV-eGFP vector only or CMV-eGFP plus nontargeting siRNAs. The percentage of GFP ${ }^{+}$cells positive for early (MBP) or late (MOG) myelin gene expression was assessed for each condition. Data were normalized to control levels of MBP or MOG expression. Average control MBP ${ }^{+}$cells, $83.8 \%, \mathrm{MOG}^{+}$ cells, $60.2 \%$. All control-normalized data were averaged and presented \pm SEM; control-MBP, $n=15 ;$ siSOX10-MBP, $n=9$; siZFP536-MBP, $n=3$; CMV-UHRF1-MBP, $n=3$; control-MOG, $n=15 ;$ sisOX10-MOG, $n=6$; siZFP536, $n=3$; CMV-UHRF1-MOG, $n=6 .{ }^{*} p<0.01$, post hoc Holm-Sidak test compared with control.

protein (MAG), and myelin oligodendrocyte basic protein (MOBP) (Baumann and Pham-Dinh, 2001). The remaining 10 genes are all very likely important for normal OL function, because they were as highly upregulated and as highly expressed as the myelin proteins. One of the most surprising of these is pancreatic lipase $(P N L I P)$. Although PNLIP has been thought to be specific to the pancreas, CNS expression of a pancreatic-like lipase by an enzymatic assay has been previously shown by Tsujita et al. (1998). We confirmed the localization of the PNLIP protein in the myelinated optic nerve by Western blotting (supplemental Fig. S2, available at www.jneurosci.org as supplemental material). PNLIP hydrolyzes triglycerides into fatty acids in the digestive system (Lowe et al., 1989), and may therefore provide a source of fatty acids incorporated into membranes during myelination. Interestingly, pancreatic lipase release during pancreatitis is implicated in pancreatic encephalopathy demyelination (Estrada et al., 1979), raising the question of whether OL PNLIP misregulation could be involved in other demyelinating diseases such as MS.
Three of the identified OL-specific genes, two of which encode transmembrane proteins, are particularly interesting candidates for roles in mediating myelination. The high level of expression and upregulation of septin 5 (SEPT5) is of particular interest, because septins play important roles in directing polarized addition of membrane (Beites et al., 2005), suggesting that septin proteins may play a crucial role in myelination. Transmembrane protein 10 (TMEM10) is a mostly uncharacterized protein that is highly enriched in white matter and whose gene is upregulated 24-fold during OL differentiation (Table 1), making it a novel candidate CNS myelin protein. Finally, we also detected myelin protein zero like 1 (MPZL1) as being highly expressed in mature OLs, both in vitro and especially in vivo (Table 2; supplemental Table S2, available at www.jneurosci.org as supplemental material). MPZL1 was identified via its functional interaction with the tyrosine phosphatase SHP-2, and found to share a $46 \%$ sequence identity with the extracellular region of P0 (Zhao and Zhao, 1998), which is an integral component of peripheral myelin. The potential roles of these proteins in CNS myelination are interesting questions for future investigation.

\section{Analysis of the genes most highly expressed by OPCs}

To identify genes specific to immature OPCs, we focused on the 50 genes most strongly repressed during OL differentiation (Table 3), and also on the 50 genes that were most strongly expressed specifically by OPCs (Table 4). As was the case with the list of most strongly expressed OL genes, we chose the 50 highest expressed genes at the OPC time point from within our set of highly regulated genes (supplemental Table S2, available at www. jneurosci.org as supplemental material), and also excluded upregulated genes, to generate a list of genes specifically enriched in the immature OPCs. Because we were focusing on genes expressed in the OPCs, we did not exclude genes with $2 \mathrm{~A}$ expression patterns; genes induced in 2As but still very strongly expressed in the original pure OPC population could be important to normal OPC function. Only 11 of 89 genes identified in this way are present on both of these OPC-specific gene lists.

Perinatal OPCs are a highly proliferative cell type, and therefore not surprisingly 25\% of the OPC-enriched genes encoded cell cycle or DNA synthesis proteins (Tables 3, 4). OPCs are also known to be highly migratory cells in vitro and in vivo. We have identified a set of highly expressed OPC-specific genes that are likely to produce proteins involved in regulating cellular motility. These include reelin, the microtubule-destabilizing protein STMN3 (Curmi et al., 1999), the CDC42-interacting protein FNBP1L (Ho et al., 2004), and the actin-interacting motor protein TPM4 (Helfman et al., 1999). ANIA4, one of the most 
strongly expressed OPC-specific genes, is a close homolog of doublecortin and CaM kinase-like 1, which effects microtubule polymerization and has been detected in migrating neuronal cells (Lin et al., 2000). One of the most unexpected findings is that OPCs express high levels of several different neuropeptide genes, including secretogranin II, chromogranin $B, V G F$, and galanin, suggesting that in addition to serving as OL precursors they are highly secretory cells. In light of the fact that OPCs have recently been shown to receive functional synapses from neurons (Lin and Bergles, 2004), the finding of high levels of secreted peptides within OPCs intriguingly suggests that these cells may release these peptides on receiving synaptic input. Another interesting finding is the detection of neuroglycan $C$ gene expression, which has been recently characterized as a novel ErbB3/ErbB2 ligand (Kinugasa et al., 2004). This is interesting, because ErbB2 activation enhances OL differentiation and survival (Fernandez et al., 2000; Park et al., 2001; Kim et al., 2003), suggesting the possibility that, in the early stages of OL differentiation, OPC-derived neuroglycan C could provide support for newly forming OLs until they tightly wrap axons, whereupon the axonally expressed ErbB2 ligand neuregulin could then support myelinating OLs (Barres and Raff, 1999).

\section{Association of genes regulated during oligodendrocyte differentiation to MS-linked genetic loci}

Several diseases in humans disrupt normal CNS myelin, such as Canavan's disease, Pelizaeus-Merzbacher disease, and multiple sclerosis (Schiffmann and Boespflug-Tanguy, 2001; Franklin, 2002; Surendran et al., 2003). Although gene mutations that cause the first two of these have been identified, the gene loci that govern MS susceptibility are still unknown. To determine whether any of the genes we have identified could be linked to genomic loci associated with demyelinating disorders, we first determined the closest mouse and human homologs and corresponding genomic loci for each of our identified highly regulated OL genes (supplemental Table S2, available at www.jneurosci.org as supplemental material). This list can therefore be consulted when a genomic region of interest associated with myelin disruption has been characterized; genes linked to such loci that are regulated during OL differentiation would merit a higher degree of initial scrutiny. In collaboration with the Popko Laboratory, we have already used this list to identify a novel myelindisrupting mutant allele of the aspartoacylase gene (ASPA) isolated in a forward genetic screen (M. Traka, R. L. Wollmann, J. C. Dugas, S. R. Cerda, B. A. Barres, and B. Popko, unpublished observation).

By correlating known MS-linked loci to genes highly regulated during OL differentiation, we next generated a list of positional candidate genes potentially involved in MS susceptibility. Fernald et al. (2005) have performed a comprehensive analysis of 29 published linkage analyses for human cases of MS. By identifying chromosomal regions that have been independently identified in three or more previous studies, they have generated a list of loci highly likely to be involved in inherited increased risk of MS.
Shown in Table 5 are the genes identified in our time course experiment that lie within $2 \mathrm{Mb}$ of these loci and are thus good candidates for genes that may help determine MS susceptibility. Some of these genes, such as MOG and CRYAB, have been previously implicated as causative antigens in MS (van Noort et al., 1995; van Veen et al., 2003; O'Connor et al., 2005). Others represent novel candidate genes whose potential causative roles in MS can be investigated in future studies.

\section{Discussion}

\section{OL differentiation progresses fully in the absence of}

\section{heterologous cell-cell interactions}

We have taken advantage of our ability to purify and stimulate the differentiation of primary mammalian OPCs to complete the first detailed genomic analysis of OL differentiation. By working with a pure population of primary OPCs, we were able to characterize with fine temporal resolution the cell-autonomous gene expression changes that produce a mature OL from a committed precursor cell. This allowed us to conclude that the cessation of OPC cell division and subsequent terminal OL differentiation is actually a complex, multistep process, which requires several days to reach completion. It has been widely demonstrated that external stimuli from axons and other sources can affect OL survival, differentiation, and myelin sheath formation (Barres et al., 1993, 1994; Barres and Raff, 1999; Stevens et al., 2002; Kim et al., 2003), but to our surprise we found little evidence for an axonal role in controlling OL gene expression. To determine how closely our in vitro study recapitulates normal OL differentiation, we have compared the gene expression profile of our in vitro-generated OLs to that of acutely isolated OLs that have matured in vivo. We found the two populations of cells to be remarkably similar, indicating that mitogen withdrawal and T3 exposure can promote full OL differentiation in the absence of heterologous cell-cell interactions. Therefore, although OLs rely on external cues for trophic support and coordinating the initiation of differentiation, the complex series of molecular events that produce a mature OL after the termination of OPC division appears to be intrinsically programmed. Once this intrinsic program has reached its com- 
Table 3. Top 50 downregulated genes during $0 \mathrm{~L}$ differentiation

\begin{tabular}{|c|c|c|c|c|}
\hline Probe set ID & Fold change & Peak level & Gene name & Gene symbol \\
\hline M93669_at & -49.52 & 1827 & Secretogranin II & SCG2 \\
\hline J03627_at & -37.01 & 1855 & S100 calcium binding protein A10 & S100A10 \\
\hline rC_AA899590_at & -27.47 & 4001 & ESTs, no homologies found & \\
\hline rC_AA899854_at & -25.81 & 3481 & Topoisomerase (DNA) $2 \alpha$ & TOP2A \\
\hline rc_AA997800_at & -22.01 & 2285 & Antigen Ki-67 (predicted) & Ki-67 \\
\hline rC_Al228113_s_at & -19.03 & 591 & Neuronal pentraxin receptor & NPTXR \\
\hline rc_Al176963_at & -18.13 & 840 & Cbp/p300-interacting transactivator 2 & CITED2 \\
\hline J03624_at & -18.00 & 2821 & Galanin & GALN \\
\hline rC_AA963443_at & -18.00 & 996 & Cyclin D2 & CCND2 \\
\hline J02585_at & -17.27 & 5693 & Stearoyl-coenzyme A desaturase 1 & SCD1 \\
\hline rC_AA956638_at & -16.45 & 525 & Ubiquitin-like, PHD + RING finger domains 1 & UHRF1 \\
\hline rC_AA944180_at & -15.89 & 1429 & CDC28 protein kinase regulatory subunit 2 & CKS2 \\
\hline rC_AA818744_at & -15.56 & 516 & Complement comp. 1, q subcomp., receptor 1 & CD93 \\
\hline rc_Al101009_at & -14.22 & 1223 & Zinc finger, CCHC domain containing 12 & ZCCHC12 \\
\hline rc_Al010123_at & -14.12 & 1384 & Transforming, acidic coiled-coil cont. protein 2 & TACC2 \\
\hline X59864mRNA_g_at & -13.83 & 3102 & H19 fetal liver mRNA & H19 \\
\hline rC_Al231702_at & -13.74 & 341 & Centrosomal protein $152 \mathrm{kDa}$ & CEP152 \\
\hline rC_AA819103_at & -13.45 & 685 & Mesoderm specific transcript homolog & MEST \\
\hline rC_AA997385_at & -13.36 & 887 & Ubiquitin specific protease 43 & USP43 \\
\hline rC_Al179576_s_at & -13.27 & 3573 & Hemoglobin, $\boldsymbol{\beta}$ & $H B B$ \\
\hline X60767mRNA_s_at & -13.09 & 1163 & Cell division cycle control protein 2 & $C D C 2$ \\
\hline rc_Al071227_at & -13.00 & 418 & DNA repair protein RAD51 homolog 1 & RAD51 \\
\hline rc_Al639088_s_at & -12.73 & 792 & ESTs, no homologies found & \\
\hline rc_AA859341_at & -12.38 & 475 & SH3 and $P X$ domains $2 A$ & SH3PXD2A \\
\hline U17565_g_at & -12.04 & 3466 & Mini chromosome maintenance deficient 6 & MCM6 \\
\hline rC_Al058975_at & -11.96 & 1063 & SRY-box containing gene 11 & S0X11 \\
\hline rC_Al146172_at & -11.88 & 1565 & Neuron specific gene family member 2 & NSG2 \\
\hline rC_AA900746_at & -11.88 & 485 & ESTs, no homologies found & \\
\hline M74223_at & -11.79 & 5120 & VGF nerve growth factor inducible & VGF \\
\hline rc_Al103150_at & -11.71 & 934 & Ubiquitin-conjugating enzyme E2C & UBE2C \\
\hline rC_Al010612_at & -11.47 & 254 & Tenascin C & TNC \\
\hline rC_AA956688_at & -11.39 & 2048 & Cell division cycle associated 3 & $C D C A 3$ \\
\hline rc_Al231497_at & -11.31 & 1171 & Karyopherin $\alpha 2$ & KPNA2 \\
\hline rc_AA893717_at & -11.24 & 2174 & Rac GTPase-activating protein 1 & RACGAP1 \\
\hline rc_Al012221_at & -11.16 & 1413 & Chloride intracellular channel 1 & CLIC1 \\
\hline rC_Al169327_g_at & -11.08 & 957 & Tissue inhibitor of metalloproteinase 1 & TIMP1 \\
\hline rc_Al045594_at & -10.93 & 1752 & Spindle pole body component 25 & $S P B C 25$ \\
\hline rc_Al103106_at & -10.78 & 1783 & Lamin B1 & LMNB1 \\
\hline rC_Al072698_at & -10.70 & 869 & TRAF4 associated factor 1 & TRAF4AF1 \\
\hline rC_AA851392_at & -10.63 & 537 & Kinesin family member 22 & KIF22 \\
\hline rC_AA818016_at & -10.56 & 2069 & Cell division cycle 23 & $C D C 23$ \\
\hline rc_Al237713_at & -10.56 & 1007 & ESTs, no homologies found & \\
\hline rC_Al103682_at & -10.56 & 307 & Polymerase (DNA directed), $\alpha 1$ & POLA \\
\hline rC_Al232326_at & -10.48 & 989 & ESTS, no homologies found & \\
\hline rC_Al145123_at & -10.48 & 989 & Similar to ataxin 2-binding protein 1 isoform 2 & \\
\hline rC_AA925012_at & -10.48 & 1711 & Geminin & GMNN \\
\hline rc_Al104821_at & -10.34 & 460 & Leucine rich repeat interacting protein 1 & LRRFIP1 \\
\hline AF019974_at & -10.27 & 381 & Chromogranin $B$ & $C H G B$ \\
\hline rC_AA943886_at & -10.20 & 830 & Polo-like kinase 2 & PLK2 \\
\hline rc_Al230215_at & -10.06 & 1336 & Cell division cycle associated 8 & $C D C A 8$ \\
\hline
\end{tabular}

The 50 unique genes most strongly repressed during $0 \mathrm{~L}$ differentiation are listed. Note that genes with a likely $2 \mathrm{~A}$ expression pattern are not included. Genes listed in bold are present in both Tables 3 and 4 . In cases in which more than one Affymetrix probe set (Probe set ID) corresponding to the same gene was highly regulated, only the most strongly regulated is listed. Fold change, Highest expression level/lowest expression level in time course; negative numbers are downregulated relative to $\mathrm{OPCS}$. Peak level, Highest raw level of expression seen at any time point in the gene chip experiment.

pletion, axons may regulate myelin formation by triggering the exocytosis of expressed, endosomally stored myelin proteins (Trajkovic et al., 2006). The data set that we have generated is fully available for all investigators (supplemental Table S1, available at www.jneurosci.org as supplemental material) and should serve as a useful data set for many future investigations.

Terminal OL differentiation is a sequential multistep process It is already appreciated that the generation of a myelinating OL occurs in several well defined differentiation stages. First, multipotent neural stem cells generate committed OPCs, after which
OPCs generate terminally differentiated, myelinating OLs. Our findings demonstrate that sequential stages of cell differentiation continue to occur during the postmitotic stages of OL differentiation. There are previous observations consistent with these data. For instance, CNP1 and GalC (the major myelin sphingolipid produced by UGT8) are known markers of early OL differentiation, whereas MOG and MOBP accumulate only in very mature OLs (Holz and Schwab, 1997; Baumann and Pham-Dinh, 2001). Our data extend this previous work by showing that there are at least two distinct sequential stages of postmitotic OL differentiation. We have better defined the OL genes that are expressed early 
Table 4. Top 50 OPC-specific expressed genes

\begin{tabular}{|c|c|c|c|c|}
\hline Probe set ID & OPC level & Fold change & Gene name & Gene symbol \\
\hline AF023087_s_at & 10,328 & -6.36 & Early growth response 1 & EGR1 \\
\hline AF030089UTR\#1_at & 8350 & -5.82 & Activity and neurotrans.-induced early gene 4 & ANIA4 \\
\hline rC_AA955914_s_at & 7687 & -4.76 & Fibrillarin & $F B L$ \\
\hline rC_AI235043_f_at & 5200 & -5.58 & Ubiquitin carboxyl-terminal hydrolase L1 & UCHL1 \\
\hline M74223_at & 5120 & -11.79 & VGF nerve growth factor inducible & VGF \\
\hline rC_AA943363_at & 4284 & -6.92 & Adenylate cyclase activ. polypep. 1 rec. type I & ADCYAP1R1 \\
\hline rC_Al639082_s_at & 4249 & -9.19 & Mini chromosome maintenance deficient 6 & MCM6 \\
\hline rC_AA964562_at & 4034 & -4.66 & ESTs, no homologies found & \\
\hline rc_AA899649_at & 3766 & -5.82 & Stathmin-like 3 & STMN3 \\
\hline X62952_at & 3693 & -25.11 & Vimentin* & VIM \\
\hline D14014_g_at & 3686 & -5.86 & Cyclin D1 & CCND1 \\
\hline X07944exon\#1-12_s_at & 3418 & -5.17 & Ornithine decarboxylase 1 & ODC1 \\
\hline rC_AA851497_f_at & 3234 & -4.35 & Hemoglobin, $\alpha 1$ & HBA1 \\
\hline L25387_g_at & 3120 & -5.70 & Phosphofructokinase, liver, B-type & PFKL \\
\hline rc_AA893471_s_at & 3062 & -7.26 & Reelin & RELN \\
\hline J03624_at & 2821 & -18.00 & Galanin & GALN \\
\hline M81642_at & 2779 & -4.92 & Coagulation factor II (thrombin) receptor & $F 2 R$ \\
\hline rc_AA892791_at & 2776 & -4.86 & Excision repair cross-complementing 1 & ERCC1 \\
\hline rC_Al179576_s_at & 2506 & -13.27 & Hemoglobin, $\boldsymbol{\beta}$ & $H B B$ \\
\hline rc_AA945604_at & 2501 & -4.14 & Dimethylarginine dimethylaminohydrolase 1 & DDAH1 \\
\hline U64030_at & 2420 & -8.28 & Deoxyuridinetriphosphatase (dUTPase) & DUTP \\
\hline rc_Al008396_at & 2374 & -5.74 & Uridine monophosphate synthetase & UMPS \\
\hline rc_AA899106_at & 2333 & -9.85 & Cyclin D2 & CCND2 \\
\hline rC_Al013911_at & 2307 & -6.63 & RNA binding motif (RNP1, RRM) protein 3 & RBM3 \\
\hline rc_Al069958_at & 2297 & -5.21 & Zinc finger, CCHC domain containing 12 & ZCCHC12 \\
\hline L21192_at & 2253 & -5.82 & Growth associated protein 43 & GAP43 \\
\hline rC_AA945679_s_at & 2238 & -7.89 & ESTs, no homologies found & \\
\hline rC_Al179610_at & 2215 & -4.50 & Heme oxygenase & HMOX1 \\
\hline AF069782_at & 2205 & -5.78 & Nucleolar protein 5 & NOL5 \\
\hline rC_AA899590_at & 2201 & -27.47 & ESTs, no homologies found & \\
\hline rC_AA901183_at & 2195 & -4.06 & Rat 455 rDNA gene transcrip. initiation region & \\
\hline rc_AA850706_at & 2045 & -8.11 & SPARC related modular calcium binding 1 & SMOC1 \\
\hline J03627_at & 1855 & -37.01 & S100 calcium binding protein A10 & S100A10 \\
\hline M93669_at & 1827 & -49.52 & Secretogranin II & SCG2 \\
\hline rc_Al014091_at & 1812 & -17.88 & Cbp/p300-interacting transactivator & CITED2 \\
\hline X70871_at & 1806 & -8.17 & Cyclin $G 1^{*}$ & CCNG1 \\
\hline rC_Al112758_at & 1768 & -4.50 & Kinesin family member $21 B$ & KIF21B \\
\hline rc_AA858636_at & 1736 & -4.92 & Mini chromosome maintenance deficient 7 & MCM7 \\
\hline D38222_s_at & 1735 & -4.17 & Protein tyrosine phosphatase, receptor type, $N$ & PTPRN \\
\hline rC_AA924772_at & 1719 & -7.26 & Metallothionein 3 & MT3 \\
\hline rC_Al008836_s_at & 1712 & -6.82 & High mobility group box 2 & HMGB2 \\
\hline rC_AA945835_at & 1700 & -5.24 & Formin binding protein 1-like & FNBP1L \\
\hline U33553_at & 1686 & -5.74 & Neuroglycan C & CSPG5 \\
\hline rc_AA859088_at & 1624 & -25.81 & Synaptotagmin $4^{*}$ & SYT4 \\
\hline rC_AA899854_at & 1589 & -25.81 & Topoisomerase (DNA) $2 \alpha$ & TOP2A \\
\hline X59864mRNA_g_at & 1582 & -13.83 & H19 fetal liver mRNA & H19 \\
\hline rC_Al230284_at & 1566 & -4.11 & Tropomycin 4 & TPM4 \\
\hline M91652compl_seq_g_at & 1534 & 7.16 & Glutamine synthetase* & GLUL \\
\hline rc_Al101481_at & 1460 & -4.14 & CaM kinase $/ / \beta$ & $C A M K 2 B$ \\
\hline rc_AA943506_at & 1451 & -4.38 & Sprouty-related, EVH1 domain containing 2 & SPRED2 \\
\hline
\end{tabular}

The 50 unique genes most strongly expressed specifically in undifferentiated OPCs are listed. Genes listed in bold are present in both Tables 3 and 4 . In cases in which more than one Affymetrix probe set (Probe set ID) corresponding to the same gene was highly expressed, only the most strongly expressed is listed. OPC level, Raw expression level at the OPC time point. Fold change, Highest expression level/lowest expression level in time course; negative numbers are downregulated relative to OPCs. Genes that show a 2 A expression pattern are denoted by an asterisk (*). Note that M81225_at (FNTA, 2103 at OPC time point), rC_AI233362_at (LAMP1, 1911 at OPC), rC_AA848813_at (REEP5, 1851 at OPC), and rc_Al102031_at (BIN1, 1687 at OPC) were excluded because they are more highly expressed in mature 0Ls.

and late during differentiation, and also identified concordantly regulated transcription factors that likely control the expression of OL genes at these distinct stages. Some of the highest-level myelin genes that fall into the first stage of OL differentiation are UGT8, CNP1, MBP, PLP, OSP, CD9, EDG2, ENPP2, PNLIP, and SEPT5. Some of the highest-level myelin (and related) genes that fall into the later-induced second group are $M A G, M O G, M O B P$, MAL, ASPA, Tetraspanin 2, TM4SF11/plasmalipin, CD81, and transferrin. These distinct stages of OL differentiation are also observed in vivo; when acutely isolated newly differentiating
$\mathrm{GalC}^{+} \mathrm{MOG}^{-}$OLs are segregated from fully mature, myelinating $\mathrm{MOG}^{+}$OLs (Solly et al., 1996), the relative levels of gene expression in these two populations predominantly mimic our temporal in vitro observations (B. Emery and B. A. Barres, unpublished observations). These different waves of gene expression are not limited to the myelin genes, and in fact distinct temporal patterns of expression are seen for genes of all functional classes.

These findings raise an important question: Why are there distinct sequential stages of terminal OL differentiation? Our un- 
Table 5. Regulated genes associated with MS-linked loci

\begin{tabular}{|c|c|c|c|c|c|c|c|}
\hline Probe set ID & Peak level & Fold change & r-UniGene & h-UniGene & h-Chromosome & Gene name & $\overline{\text { Gene symbol }}$ \\
\hline rC_AA944463_at & 294 & -3.86 & Rn.7736 & Hs. 471200 & $2 q 33.3 ; 206 \mathrm{Mb}$ & Neuropilin 2 & NRP2 \\
\hline rC_Al230247_s_at & 606 & 22.32 & Rn.1451 & Hs.275775 & $5 p 12 ; 43 \mathrm{Mb}$ & Selenoprotein P, plasma, 1 & SEPP1 \\
\hline rC_AA893970_at & 1425 & 4.26 & Rn.12956 & Hs.407926 & $5 p 13.1 ; 39 \mathrm{Mb}$ & TORC2-specific protein AV03 & RICTOR \\
\hline X62875mRNA_g_at & 731 & -4.47 & Rn.83614 & Hs.518805 & $6 p 21.31 ; 34 \mathrm{Mb}$ & High mobility group AT-hook 1 & HMGa1 \\
\hline rC_Al229707_s_at & 727 & -6.11 & Rn.2458 & Hs.533059 & $6 p 21.33 ; 31 \mathrm{Mb}$ & Tubulin $\beta 5$ chain & TUBB \\
\hline rC_Al012221_at & 1413 & -11.16 & Rn.101003 & Hs.414565 & $6 \mathrm{p} 21.33 ; 32 \mathrm{Mb}$ & Chloride intracellular channel 1 & CLIC1 \\
\hline M99485_at & 3636 & 97.68 & Rn.9687 & Hs.141308 & $6 \mathrm{p} 22.1 ; 30 \mathrm{Mb}$ & Myelin oligodendrocyte glycoprotein & MOG \\
\hline rC_AA925012_at & 1711 & -10.48 & Rn.15438 & Hs.234896 & $6 \mathrm{p} 22.2 ; 25 \mathrm{Mb}$ & Geminin & GMNN \\
\hline rC_Al169703_at & 581 & -3.78 & Rn.137382 & Hs.484813 & $6 \mathrm{p} 22.3 ; 18 \mathrm{Mb}$ & DEK oncogene (DNA binding) & DEK \\
\hline rC_AA891734_at & 2285 & 7.46 & Rn.54151 & Hs.520814 & $7 \mathrm{p} 12.3 ; 47 \mathrm{Mb}$ & Tensin-like SH2 domain containing 1 & TENS1 \\
\hline rc_AA998383_at & 424 & -6.28 & Rn.98822 & Hs.119882 & $7 q 21.2 ; 92 \mathrm{Mb}$ & Cyclin-dependent kinase 6 & CDK6 \\
\hline rC_Al233394_at & 119 & 7.62 & Rn.8331 & Hs.116796 & $11 \mathrm{q} 23.1 ; 111 \mathrm{Mb}$ & DIX domain containing 1 & DIXDC1 \\
\hline M55534mRNA_s_at & 638 & 5.13 & Rn.98208 & Hs.408767 & $11 \mathrm{q} 23.1 ; 111 \mathrm{Mb}$ & Crystallin, $\alpha B$ & $C R Y A B$ \\
\hline rC_Al232128_at & 901 & 5.46 & Rn.19811 & Hs.47115 & $16 q 22.2 ; 70 \mathrm{Mb}$ & Hydrocephalus inducing & HYDIN \\
\hline rc_Al073086_at & 1031 & 3.68 & Rn.17753 & Hs.531704 & $17 q 24.2 ; 62 \mathrm{Mb}$ & Protein kinase $C, \alpha$ & PRKCA \\
\hline rC_Al231497_at & 1171 & -11.31 & Rn.2949 & Hs.159557 & $17 q 24.2 ; 63 \mathrm{Mb}$ & Karyopherin $\alpha 2$ & KPNA2 \\
\hline rC_Al045866_at & 349 & -3.76 & Rn.16334 & Hs.549130 & $17 q 24.2 ; 63 \mathrm{Mb}$ & PI transfer protein, cytoplasmic 1 & PITPNC1 \\
\hline rC_Al233288_at & 493 & 7.46 & Rn.19813 & Hs.463964 & $17 q 24.2 ; 64 \mathrm{Mb}$ & WD40 repeat protein Interact with PI & WIPI49 \\
\hline rC_Al009456_at & 1539 & 13.74 & Rn.22789 & Hs.58351 & $17 q 24.2 ; 64 \mathrm{Mb}$ & ATP-binding cassette transporter $8 a$ & $A B C A 8$ \\
\hline rC_AA818774_at & 1368 & 12.13 & Rn.16666 & Hs.27935 & $17 q 25.1 ; 70 \mathrm{Mb}$ & Tweety homolog 2 & TTYH2 \\
\hline rC_Al230702_at & 910 & -5.13 & Rn.104570 & Hs.532803 & $17 q 25.1 ; 71 \mathrm{Mb}$ & Hematological and neurological EST 1 & HN1 \\
\hline rC_Al012571_at & 535 & -4.99 & Rn.12319 & Hs.501423 & 19p13.3; $3 \mathrm{Mb}$ & Bruno-like 5, RNA binding protein & BRUNOL5 \\
\hline
\end{tabular}

Genes regulated during $0 \mathrm{~L}$ differentiation tightly linked to multiple sclerosis susceptibility loci. Genes highly regulated during $0 \mathrm{~L}$ differentiation that lay within $2 \mathrm{Mb}$ of human genomic loci linked to cases of multiple sclerosis in three or more independent clinical studies (Fernald et al., 2005). Peak level, Highest raw expression level reached in the time course. Fold change, Highest expression level/lowest expression level in time course; if highest expression level precedes lowest expression level, expressed as negative fold change. $r$ - and h-UniGene, respectively, The rat and closest human homolog UniGene clusters associated with the indicated probe set. h-Chromosome, The cytoband and location in megabases from the end of the p-arm for each listed human UniGene cluster.

published time-lapse studies of CNS myelination (T. A. Watkins and B. A. Barres, unpublished observations) clearly demonstrate at least two morphological stages of OL differentiation. After an $\mathrm{OL}$ is generated, it must first extend processes along axons, selecting which axons it will align with and ensheathe, and only subsequently does it myelinate these axons. The two waves of myelin gene expression we observe may correspond to these two stages of morphological differentiation. In fact, in culture, even in the absence of axons, the first few days of OL differentiation are characterized by robust process outgrowth, whereas the last few days are associated with membranous elaboration from these processes (Fig. 1A). Consistent with this possibility, CNP1, which is induced early in OL differentiation, is involved in regulating microtubule-based cytoskeletal changes (Lee et al., 1995). According to this model, the synchronous gene changes invoked when an OL begins to differentiate may act as a built-in timer, providing the OL several days to find and ensheathe axons, at which time myelination may be triggered. The successive stages of gene expression during OL differentiation also raises the question of whether an OL that has myelinated can ever myelinate again, for instance after myelin is lost in demyelinating diseases such as MS. Quite possibly, an OL can only play out this intrinsic program once in its lifetime.

Last, we have shown that myelin genes expressed in successive stages of OL terminal differentiation are differentially controlled by distinct, coordinately regulated transcription factors. These data provide evidence for the existence of distinct functional programs driving the successive stages of OL differentiation. Our identification of genes that are able to control different stages of OL gene expression will allow us to determine the functional consequences of selectively disrupting later stages of OL gene expression in future experiments. Furthermore, because these two stages of OL differentiation can be controlled independently, it might be possible under pathophysiological circumstances for the first stage of OL differentiation to occur but for the subsequent stage to be inhibited. This raises the question of whether the failure of remyelination to occur in MS plaques, even when surviving OLs are present and contacting axons (Lucchinetti et al., 1999), could be attributable to such a halt in OL differentiation. Using the specific markers of early and late OL differentiation that we have identified, this possibility can now be tested. If so, a more complete understanding of how the later stage of OL differentiation is controlled may reveal novel ways in which to promote remyelination.

\section{Identification of novel candidate genes that may intrinsically control OL differentiation}

We identified a number of transcription factor genes that are dynamically regulated during OL differentiation. We have demonstrated that two of these, UHRF1 and ZFP536, genes previously unstudied in OLs, specifically regulate genes expressed in the later phase of OL differentiation. These findings demonstrate the utility of genomic expression analysis in identifying novel regulators of OL differentiation, and we anticipate that several other transcription factors identified here will also be found to be playing important roles in generating mature OLs.

Another interesting candidate for regulating OL differentiation is CDKN1C. CDKN1C (also known as $p 57^{K I P 2}$ ), which is induced by $\mathrm{p} 73$, is one of the earliest and most rapidly upregulated genes during OL differentiation, and is a powerful intracellular inhibitor of cell proliferation (Lee et al., 1995; Blint et al., 2002). A recent study has determined that CDKN1C is required in zebrafish for neural progenitor cells to stop dividing and generate OPCs, and thus for normal OL generation (Park et al., 2005). Our finding that $C D K N 1 C$ is upregulated as OPCs differentiate into OLs suggests a potentially important role in inducing terminal OL differentiation as well.

The transcription factors we have identified as highly regulated in distinct temporal waves during OL differentiation are excellent new candidates for controlling this complex process, and we have begun to assay these various genes for their ability to regulate OL differentiation. Fully characterizing the regulation of 
terminal OL differentiation may lead to a greater understanding of how this process is disrupted in leukoencephalopathies such as MS, and may also enhance our ability to specify pools of potentially remyelinating cells from uncommitted pools of precursor cells for cellular replacement therapies.

\section{Identification of new candidate genes that may govern MS susceptibility}

We identified several new genes expressed by OLs that are potential candidates for influencing MS susceptibility (Table 5). Perhaps the most interesting potential MS candidate newly identified here is SEPP1, which is upregulated by $>20$-fold as OLs differentiate, making it one of the most strongly induced OL genes (Table 1). Moreover, it is highly enriched in adult white matter by in situ hybridization (Allen Brain Atlas). SEPP1 is required to maintain normal selenium levels in several tissues, including the CNS (Hill et al., 2003), and deficiencies in SEPP1 lead to neurological defects (Schweizer et al., 2004). Although selenium and SEPP1 have been found to be strongly neurotrophic (Yan and Barrett, 1998), these effects may also partially be mediated by disruptions in normal OL development: selenium is required for the survival and differentiation of oligodendrocyte lineage cells in vitro (Bottenstein, 1986), and for myelin gene upregulation (Gu et al., 1997). In addition, there have been suggestions that environmental deficits in selenium could be a contributing risk factor for MS (Hasanen et al., 1986; Irvine et al., 1989) and that selenium supplementation might be beneficial (Clausen et al., 1988). These factors all combine to make SEPP1 a strong candidate for the MS susceptibility gene located at $5 \mathrm{p} 12$ 13. Future studies will be important to determine the relevance of these new candidate genes in MS.

\section{References}

Arima Y, Hirota T, Bronner C, Mousli M, Fujiwara T, Niwa S, Ishikawa H, Saya H (2004) Down-regulation of nuclear protein ICBP90 by p53/ p21Cip1/WAF1-dependent DNA-damage checkpoint signals contributes to cell cycle arrest at $\mathrm{G}_{1} / \mathrm{S}$ transition. Genes Cells 9:131-142.

Arnett HA, Wang Y, Matsushima GK, Suzuki K, Ting JP (2003) Functional genomic analysis of remyelination reveals importance of inflammation in oligodendrocyte regeneration. J Neurosci 23:9824-9832.

Barres BA, Raff MC (1999) Axonal control of oligodendrocyte development. J Cell Biol 147:1123-1128.

Barres BA, Hart IK, Coles HS, Burne JF, Voyvodic JT, Richardson WD, Raff MC (1992) Cell death and control of cell survival in the oligodendrocyte lineage. Cell 70:31-46.

Barres BA, Schmid R, Sendnter M, Raff MC (1993) Multiple extracellular signals are required for long-term oligodendrocyte survival. Development 118:283-295.

Barres BA, Lazar MA, Raff MC (1994) A novel role for thyroid hormone, glucocorticoids and retinoic acid in timing oligodendrocyte development. Development 120:1097-1108.

Baumann N, Pham-Dinh D (2001) Biology of oligodendrocyte and myelin in the mammalian central nervous system. Physiol Rev 81:871-927.

Beites CL, Campbell KA, Trimble WS (2005) The septin Sept5/CDCrel-1 competes with alpha-SNAP for binding to the SNARE complex. Biochem J 385:347-353.

Blint E, Phillips AC, Kozlov S, Stewart CL, Vousden KH (2002) Induction of p57(KIP2) expression by p73beta. Proc Natl Acad Sci USA 99:3529-3534.

Booher RN, Holman PS, Fattaey A (1997) Human Myt1 is a cell cycleregulated kinase that inhibits cdc2 but not cdk2 activity. J Biol Chem 272:22300-22306.

Bottenstein JE (1986) Growth requirements in vitro of oligodendrocyte cell lines and neonatal rat brain oligodendrocytes. Proc Natl Acad Sci USA 83:1955-1959.

Chan JR, Watkins TA, Cosgaya JM, Zhang C, Chen L, Reichardt LF, Shooter EM, Barres BA (2004) NGF controls axonal receptivity to myelination by Schwann cells or oligodendrocytes. Neuron 43:183-191.

Clausen J, Jensen GE, Nielsen SA (1988) Selenium in chronic neurologic diseases. Multiple sclerosis and Batten's disease. Biol Trace Elem Res 15:179-203.

Curmi PA, Gavet O, Charbaut E, Ozon S, Lachkar-Colmerauer S, Manceau V, Siavoshian S, Maucuer A, Sobel A (1999) Stathmin and its phosphoprotein family: general properties, biochemical and functional interaction with tubulin. Cell Struct Funct 24:345-357.

Estrada RV, Moreno J, Martinez E, Hernandez MC, Gilsanz G, Gilsanz V (1979) Pancreatic encephalopathy. Acta Neurol Scand 59:135-139.

Fernald GH, Yeh RF, Hauser SL, Oksenberg JR, Baranzini SE (2005) Mapping gene activity in complex disorders: integration of expression and genomic scans for multiple sclerosis. J Neuroimmunol 167:157-169.

Fernandez PA, Tang DG, Cheng L, Prochiantz A, Mudge AW, Raff MC (2000) Evidence that axon-derived neuregulin promotes oligodendrocyte survival in the developing rat optic nerve. Neuron 28:81-90.

Franklin RJ (2002) Why does remyelination fail in multiple sclerosis? Nat Rev Neurosci 3:705-714.

Gu J, Royland JE, Wiggins RC, Konat GW (1997) Selenium is required for normal upregulation of myelin genes in differentiating oligodendrocytes. J Neurosci Res 47:626-635.

Hasanen E, Kinnunen E, Alhonen P (1986) Relationships between the prevalence of multiple sclerosis and some physical and chemical properties of soil. Sci Total Environ 58:263-272.

Helfman DM, Berthier C, Grossman J, Leu M, Ehler E, Perriard E, Perriard JC (1999) Nonmuscle tropomyosin-4 requires coexpression with other low molecular weight isoforms for binding to thin filaments in cardiomyocytes. J Cell Sci 112:371-380.

Hill KE, Zhou J, McMahan WJ, Motley AK, Atkins JF, Gesteland RF, Burk RF (2003) Deletion of selenoprotein $P$ alters distribution of selenium in the mouse. J Biol Chem 278:13640-13646.

Ho HY, Rohatgi R, Lebensohn AM, Le M, Li J, Gygi SP, Kirschner MW (2004) Toca-1 mediates Cdc42-dependent actin nucleation by activating the N-WASP-WIP complex. Cell 118:203-216.

Holz A, Schwab ME (1997) Developmental expression of the myelin gene MOBP in the rat nervous system. J Neurocytol 26:467-477.

Hu JG, Fu SL, Zhang KH, Li Y, Yin L, Lu PH, Xu XM (2004) Differential gene expression in neural stem cells and oligodendrocyte precursor cells: a cDNA microarray analysis. J Neurosci Res 78:637-646.

Irizarry RA, Bolstad BM, Collin F, Cope LM, Hobbs B, Speed TP (2003) Summaries of Affymetrix GeneChip probe level data. Nucleic Acids Res 31:e15.

Irvine DG, Schiefer HB, Hader WJ (1989) Geotoxicology of multiple sclerosis: the Henribourg, Saskatchewan, cluster focus. I. The water. Sci Total Environ 84:45-59.

Jessen KR, Mirsky R (2005) The origin and development of glial cells in peripheral nerves. Nat Rev Neurosci 6:671-682.

Jurevics H, Largent C, Hostettler J, Sammond DW, Matsushima GK, Kleindienst A, Toews AD, Morell P (2002) Alterations in metabolism and gene expression in brain regions during cuprizone-induced demyelination and remyelination. J Neurochem 82:126-136.

Kim JY, Sun Q, Oglesbee M, Yoon SO (2003) The role of ErbB2 signaling in the onset of terminal differentiation of oligodendrocytes in vivo. J Neurosci 23:5561-5571.

Kinugasa Y, Ishiguro H, Tokita Y, Oohira A, Ohmoto H, Higashiyama S (2004) Neuroglycan C, a novel member of the neuregulin family. Biochem Biophys Res Commun 321:1045-1049.

Lee MH, Reynisdottir I, Massague J (1995) Cloning of p57KIP2, a cyclindependent kinase inhibitor with unique domain structure and tissue distribution. Genes Dev 9:639-649.

Lin PT, Gleeson JG, Corbo JC, Flanagan L, Walsh CA (2000) DCAMKL1 encodes a protein kinase with homology to doublecortin that regulates microtubule polymerization. J Neurosci 20:9152-9161.

Lin SC, Bergles DE (2004) Synaptic signaling between GABAergic interneurons and oligodendrocyte precursor cells in the hippocampus. Nat Neurosci 7:24-32.

Lowe ME, Rosenblum JL, Strauss AW (1989) Cloning and characterization of human pancreatic lipase cDNA. J Biol Chem 264:20042-20048.

Lucchinetti C, Bruck W, Parisi J, Scheithauer B, Rodriguez M, Lassmann H (1999) A quantitative analysis of oligodendrocytes in multiple sclerosis lesions. A study of 113 cases. Brain 122:2279-2295.

Mabie PC, Mehler MF, Marmur R, Papavasiliou A, Song Q, Kessler JA (1997) Bone morphogenetic proteins induce astroglial differentiation of oligodendroglial-astroglial progenitor cells. J Neurosci 17:4112-4120. 
Masahira N, Takebayashi H, Ono K, Watanabe K, Ding L, Furusho M, Ogawa Y, Nabeshima YI, Alvarez-Buylla A, Shimizu K, Ikenaka K (2006) Olig2positive progenitors in the embryonic spinal cord give rise not only to motoneurons and oligodendrocytes, but also to a subset of astrocytes and ependymal cells. Dev Biol 293:358-369.

Miller RH, David S, Patel R, Abney ER, Raff MC (1985) A quantitative immunohistochemical study of macroglial cell development in the rat optic nerve: in vivo evidence for two distinct astrocyte lineages. Dev Biol 111:35-41.

Nakamura K, Harada C, Namekata K, Harada T (2006) Expression of olig2 in retinal progenitor cells. NeuroReport 17:345-349.

Nielsen JA, Berndt JA, Hudson LD, Armstrong RC (2004) Myelin transcription factor 1 (Myt1) modulates the proliferation and differentiation of oligodendrocyte lineage cells. Mol Cell Neurosci 25:111-123.

O’Connor KC, Appel H, Bregoli L, Call ME, Catz I, Chan JA, Moore NH, Warren KG, Wong SJ, Hafler DA, Wucherpfennig KW (2005) Antibodies from inflamed central nervous system tissue recognize myelin oligodendrocyte glycoprotein. J Immunol 175:1974-1982.

Orian JM, Ahern AJ, Ayers MM, Levine JM, Tapp LD, Reynolds R (2001) Disturbed oligodendrocyte development and recovery from hypomyelination in a c-myc transgenic mouse mutant. J Neurosci Res 66:46-58.

Park HC, Boyce J, Shin J, Appel B (2005) Oligodendrocyte specification in zebrafish requires notch-regulated cyclin-dependent kinase inhibitor function. J Neurosci 25:6836-6844.

Park SK, Miller R, Krane I, Vartanian T (2001) The erbB2 gene is required for the development of terminally differentiated spinal cord oligodendrocytes. J Cell Biol 154:1245-1258.

Pati D, Keller C, Groudine M, Plon SE (1997) Reconstitution of a MEC1independent checkpoint in yeast by expression of a novel human fork head cDNA. Mol Cell Biol 17:3037-3046.

Ranscht B, Clapshaw PA, Price J, Noble M, Seifert W (1982) Development of oligodendrocytes and Schwann cells studied with a monoclonal antibody against galactocerebroside. Proc Natl Acad Sci USA 79:2709-2713.

Rowitch DH (2004) Glial specification in the vertebrate neural tube. Nat Rev Neurosci 5:409-419.

Schiffmann R, Boespflug-Tanguy O (2001) An update on the leukodsytrophies. Curr Opin Neurol 14:789-794.

Schweizer U, Brauer AU, Kohrle J, Nitsch R, Savaskan NE (2004) Selenium and brain function: a poorly recognized liaison. Brain Res Brain Res Rev 45:164-178.

Sock E, Leger H, Kuhlbrodt K, Schreiber J, Enderich J, Richter-Landsberg C, Wegner M (1997) Expression of Krox proteins during differentiation of the O-2A progenitor cell line CG-4. J Neurochem 68:1911-1919.

Solly SK, Thomas JL, Monge M, Demerens C, Lubetzki C, Gardinier MV,
Matthieu JM, Zalc B (1996) Myelin/oligodendrocyte glycoprotein (MOG) expression is associated with myelin deposition. Glia 18:39-48.

Stevens B, Porta S, Haak LL, Gallo V, Fields RD (2002) Adenosine: a neuron-glial transmitter promoting myelination in the CNS in response to action potentials. Neuron 36:855-868.

Stolt CC, Rehberg S, Ader M, Lommes P, Riethmacher D, Schachner M, Bartsch U, Wegner M (2002) Terminal differentiation of myelinforming oligodendrocytes depends on the transcription factor Sox10. Genes Dev 16:165-170.

Surendran S, Matalon KM, Tyring SK, Matalon R (2003) Molecular basis of Canavan's disease: from human to mouse. J Child Neurol 18:604-610.

Tai YC, Speed TP (2006) A multivariate empirical Bayes statistic for replicated microarray time course data. Ann Stat, in press.

Trajkovic K, Dhaunchak AS, Goncalves JT, Wenzel D, Schneider A, Bunt G, Nave KA, Simons M (2006) Neuron to glia signaling triggers myelin membrane exocytosis from endosomal storage sites. J Cell Biol 172:937-948.

Tsujita T, Sumida M, Sumiyoshi M, Kameda K, Okuda H (1998) Alkaline lipase from brain: is it the same enzyme as pancreatic lipase from pancreas? Arch Biochem Biophys 352:44-50.

van Noort JM, van Sechel AC, Bajramovic JJ, el Ouagmiri M, Polman CH, Lassmann H, Ravid R (1995) The small heat-shock protein alpha B-crystallin as candidate autoantigen in multiple sclerosis. Nature 375:798-801.

van Veen T, van Winsen L, Crusius JB, Kalkers NF, Barkhof F, Pena AS, Polman CH, Uitdehaag BM (2003) $\alpha$ B-crystallin genotype has impact on the multiple sclerosis phenotype. Neurology 61:1245-1249.

Wegner M (2001) Expression of transcription factors during oligodendroglial development. Microsc Res Tech 52:746-752.

Wu S, Wu Y, Capecchi MR (2006) Motoneurons and oligodendrocytes are sequentially generated from neural stem cells but do not appear to share common lineage-restricted progenitors in vivo. Development 133:581-590.

Yamazaki F, Nagatsuka Y, Shirakawa H, Yoshida M (1995) Repression of cell cycle progression by antisense HMG2 RNA. Biochem Biophys Res Commun 210:1045-1051.

Yan J, Barrett JN (1998) Purification from bovine serum of a survivalpromoting factor for cultured central neurons and its identification as selenoprotein-P. J Neurosci 18:8682-8691.

Zhao ZJ, Zhao R (1998) Purification and cloning of PZR, a binding protein and putative physiological substrate of tyrosine phosphatase SHP-2. J Biol Chem 273:29367-29372.

Zhou Q, Anderson DJ (2002) The bHLH transcription factors OLIG2 and OLIG1 couple neuronal and glial subtype specification. Cell 109:61-73. 